\title{
Micromechanics-Based Progressive Failure Analysis of Composite Laminates Using Different Constituent Failure Theories
}

\author{
Albert M. Moncada ${ }^{1}$ and Aditi Chattopadhyay ${ }^{2}$ \\ Arizona State University, Tempe, Arizona, 85287 \\ Brett A. Bednarcyk ${ }^{3}$ and Steven M. Arnold ${ }^{4}$ \\ NASA Glenn Research Center, Cleveland, Ohio, 44135
}

\begin{abstract}
Predicting failure in a composite can be done with ply level mechanisms and/or micro level mechanisms. This paper uses the Generalized Method of Cells and High-Fidelity Generalized Method of Cells micromechanics theories, coupled with classical lamination theory, as implemented within NASA's Micromechanics Analysis Code with Generalized Method of Cells. The code is able to implement different failure theories on the level of both the fiber and the matrix constituents within a laminate. A comparison is made among maximum stress, maximum strain, Tsai-Hill, and Tsai-Wu failure theories. To verify the failure theories the Worldwide Failure Exercise (WWFE) experiments have been used. The WWFE is a comprehensive study that covers a wide range of polymer matrix composite laminates. The numerical results indicate good correlation with the experimental results for most of the composite layups, but also point to the need for more accurate resin damage progression models.
\end{abstract}

\section{Introduction}

$\mathrm{T}$

he Worldwide Failure Exercise (WWFE) was a collection of comprehensive experiments that covered a wide range of polymer matrix composites (PMCs) [1,2]. Six laminates were used to achieve a broad range of layups. They used different types of composite materials (fiber and matrix), lay-up of laminates (unidirectional, angle-ply, cross-ply, quasi-isotropic), and loading conditions. The contributors to the exercise were given the same material data for the plies and constituents. The WWFE data was then compared with the different PMC failure theory predictions from various authors $[3,4]$. It should be noted that the WWFE was geared towards ply level failure analysis methods rather than micromechanics based methods like those employed herein. Thus, while basic fiber and matrix elastic and strength properties were provided, additional data needed to accurately capture the nonlinear neat resin response curves, were not.

In the present paper, the Generalized Method of Cells (GMC) and High-Fidelity Generalized Method of Cells (HFGMC) micromechanics theories, coupled with classical lamination theory $[5,6]$ are employed, as implemented within NASA's Micromechanics Analysis Code with Generalized Method of Cell (MAC/GMC) [7] to predict the WWFE laminate behavior. The focus of the study is a comparison among the maximum stress, maximum strain, Tsai-Hill, and Tsai-Wu failure theories, which are active on the micro (fiber/matrix) scale. It is stressed that no modifications have been made to account for the in-situ behavior of the constituents. Rather, pure predictions have been made using the constituent properties provided by the WWFE. As such, the predictions presented herein do not benefit from the "anchor points" along the axes within a ply level failure envelope plot that correspond to the ply tensile and compressive strengths. That is, in the present models, these anchor points themselves are predicted

${ }^{1}$ Graduate Research Assistant, Department of Mechanical and Aerospace Engineering, amoncada@asu.edu.

${ }^{2}$ Professor, Department of Mechanical and Aerospace Engineering, aditi@asu.edu, AIAA Fellow.

${ }^{3}$ Materials Research Engineer, 21000 Brookpark Rd., Brett.A.Bednarcyk@nasa.gov, AIAA Senior Member.

${ }^{4}$ Senior Research Engineer, 21000 Brookpark Rd.,Steven.M.Arnold@nasa.gov. 
from the constituent level elastic and strength properties. Consequently, the overall correlation with experiment would be expected to benefit from backing out in-situ properties that would enable matching of the ply level response. Furthermore, the simplest damage progression model has been employed at the fiber/matrix scale, namely, a step function. That is, once a subvolume within the micromechanics model satisfies the applicable failure criterion, that subvolume is instantaneously assigned a near zero stiffness. The predictions would benefit from a more progressive transition from damage initiation to complete loss of stiffness on the micro scale. The results presented herein should thus be interpreted as the first step in the application of GMC and HFGMC to PMC laminate failure, where the needs for improvement have been identified and are currently being addressed.

\section{Background}

The goal of micromechanics is, given the arrangement and properties/behavior of the constituent materials within a composite, to predict the properties/behavior of the composite material. If only effective elastic properties are required, the micromechanics problem simplifies considerably, and a number of micromechanics theories will provide reasonable results (see Herakovich, [5], for examples and comparisons). If, however, local nonlinear effects, such as damage, debonding, and inelasticity must be captured, the micromechanics theory must provide access to the local stress and strain fields throughout the composite. Then, for example, when the matrix at a particular location within the simulated composite reaches its yield or failure stress, a local model for the inelastic strain accumulation or damage response can be utilized. A key advantage of micromechanics vs. macromechanics of a ply is the ability to apply such nonlinear models at the constituent scale, where the material is monolithic and usually isotropic.

The family of micromechanics theories, known collectively as the Generalized Method of Cells (GMC) has been employed in the present paper. These methods provide semi-closed form expressions for the effective constitutive behavior of a composite material, including nonlinear effects such as damage, debonding, and inelasticity, which can be modeled internally based on the local fiber and matrix stress and strain fields. The original method of cells $[8,9]$, which considers a doubly periodic repeating unit cell consisting of four subcells (typically, one fiber and three matrix) was generalized by Paley and Aboudi [10] to consider an arbitrary number of subcells. The resulting GMC thus enables analysis of repeating unit cells containing more than two constituent materials, a refined circular fiber shape, and several fibers arranged in a given packing. A triply periodic version of GMC was also developed [11] which can model short fiber and particulate reinforced composites, as well as porous materials. Aboudi [12] provided a review paper describing work done to date on GMC by researchers around the world.

It is well-known that a feature limiting the accuracy of GMC's local stress and strain fields is the method's lack of shear coupling. This lack of shear coupling means that, when global loading consisting only of normal stresses and strains is applied, only local internal normal stress and strain fields are present, with no shear. Conversely, applying only global shears in GMC results in only local shears, with no normal stresses and strains. The first order displacement field assumed within the subcells causes this lack of shear coupling along with three additional important features. First, GMC is completely insensitive to subcell grid refinement. Thus, the least refined representation that allows one to capture the salient features of the microstructural geometry can be employed. Second, the stress and strain components are constant within each subcell. Thus no additional quadrature points are necessary within each subcell to track nonlinear effects; a single subcell value for each component will suffice. Finally, GMC's lack of shear coupling has enabled the method's equations to be reformulated such that unique tractions serve as the basic unknowns, which has improved the computational efficiency of the method by hundreds to thousands of times for sufficiently complex repeating unit cells $[13,14]$.

A newer but related micromechanics model, High Fidelity Generalized Method of Cells (HFGMC), has overcome GMC's lack of shear coupling $[15,16]$. This model considers geometry identical to GMC, but employs a higher-order subcell displacement field, along with additional equations required to enforce continuity and periodicity. The result is more accurate local stress and strain fields, but with more intensive computational demands. Further, HFGMC exhibits subcell grid refinement dependence (although less extreme than typical finite element code mesh refinement), along with the need to track field variables at quadrature points within each subcell to account for nonlinear inelastic effects. Aboudi [17] provided a review paper summarizing the work done to date by researchers around the world using both the GMC and HFGMC micromechanics theories. 


\section{A. Micromechanics Models}

The two micromechanics models that are employed in this study are the generalized method of cells (GMC) [10] and the high-fidelity generalized method of cells (HFGMC) $[15,16]$. The doubly periodic versions of these models, which enable analysis of continuous fiber composites such as those considered herein, both employ a repeating unit cell to represent the composite geometry. The rectangular repeating unit cell, depicted in Fig. 1, consists of an arbitrary number of rectangular subcells, denoted by the indices $(\beta \gamma)$, each of which may contain a distinct homogeneous material. The local (subcell) constitutive equation for both models is given by,

$$
\boldsymbol{\sigma}^{(\beta \gamma)}=\mathbf{C}^{(\beta \gamma)}\left(\boldsymbol{\varepsilon}^{(\beta \gamma)}-\boldsymbol{\varepsilon}^{I(\beta \gamma)}-\boldsymbol{\varepsilon}^{T(\beta \gamma)}\right)
$$

where $\boldsymbol{\sigma}^{(\beta \gamma)}$ is the vector of average subcell stresses, $\mathbf{C}^{(\beta \gamma)}$ is the subcell elastic stiffness matrix, and $\boldsymbol{\varepsilon}^{(\beta \gamma)}, \boldsymbol{\varepsilon}^{I(\beta \gamma)}$, and $\boldsymbol{\varepsilon}^{T(\beta \gamma)}$ are the vectors of average subcell total strain, inelastic strain, and thermal strain, respectively.

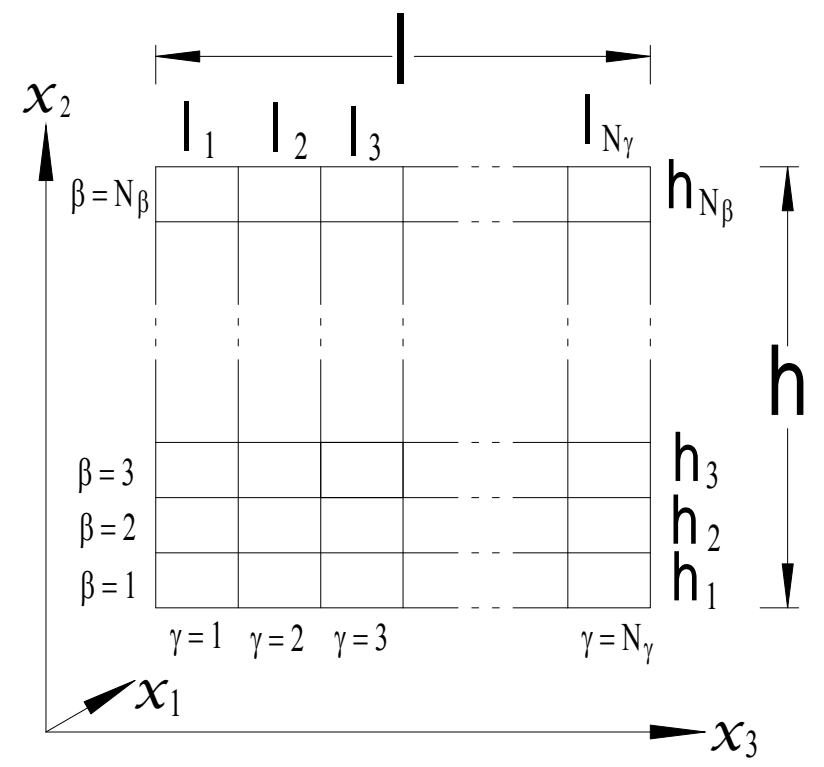

Figure 1. Repeating unit cell considered by the doubly periodic GMC and HFGMC micromechanics theories.

The basic assumption in GMC is that the displacement vector in each subcell varies linearly with the local subcell coordinates $\left(\bar{y}_{2}^{(\beta)}, \bar{y}_{3}^{(\gamma)}\right)$ located at the center of each subcell,

$$
u_{i}^{(\beta \gamma)}=W_{i(00)}^{(\beta \gamma)}+\bar{y}_{2}^{(\beta)} W_{i(10)}^{(\beta \gamma)}+\bar{y}_{3}^{(\gamma)} W_{i(01)}^{(\beta \gamma)}
$$

whereas, in HFGMC, a higher order displacement field is employed,

$$
u_{i}^{(\beta \gamma)}=\bar{\varepsilon}_{i j} x_{j}+W_{i(00)}^{(\beta \gamma)}+\bar{y}_{2}^{(\beta)} W_{i(10)}^{(\beta \gamma)}+\bar{y}_{3}^{(\gamma)} W_{i(01)}^{(\beta \gamma)}+\frac{1}{2}\left(3 \bar{y}_{2}^{(\beta) 2}-\frac{h_{\beta}^{2}}{4}\right) W_{i(20)}^{(\beta \gamma)}+\frac{1}{2}\left(3 \bar{y}_{3}^{(\gamma) 2}-\frac{l_{\gamma}^{2}}{4}\right) W_{i(02)}^{(\beta \gamma)}
$$

The GMC formulation involves imposition of continuity of displacements and tractions between adjacent subcells and repeating unit cells in an integral, or average, sense. In the original work of Paley and Aboudi [10], this 
procedure resulted in a system of $6 N_{\beta} N_{\gamma}$ linear algebraic equations in which the strains in the subcells (which are constant within each subcell) serve as the basic unknown quantities. Note that $N_{\beta}$ and $N_{\gamma}$ are the number of subcells within the repeating unit cell in the two in-plane directions (see Fig. 2). The GMC equations have been computationally optimized by Pindera and Bednarcyk [13] such that subcell stress components serve as the unknown variables, which leads to an alternative system of linear algebraic equations with only $N_{\beta}+N_{\gamma}$ equations plus additional decoupled equations (assuming at most orthotropic local subcell behavior). This reduced number of unknown quantities (e.g., degrees of freedom) provides GMC with a high level of computational efficiency. This system of equations (involving the normal stress components) can be written as,

$$
\tilde{\mathbf{G}} \mathbf{T}=\mathbf{f}^{m}-\mathbf{f}^{I}-\mathbf{f}^{T}
$$

while the additional decoupled equations (involving the shear stress components) can be written as,

$$
\tilde{G}_{12}^{(\gamma)} T_{12}^{(\gamma)}=f_{12}^{m}-f_{12}^{I(\gamma)}, \quad \tilde{G}_{13}^{(\beta)} T_{13}^{(\beta)}=f_{13}^{m}-f_{13}^{I(\beta)}, \quad \tilde{G}_{23} T_{23}=f_{23}^{m}-f_{23}^{I}
$$

where the $\tilde{\mathbf{G}}$ matrix and the $\widetilde{G}_{i j}^{(\bullet)}$ terms contain information on the subcell material elastic properties and the subcell dimensions, the $\mathbf{T}$ vector and the $T_{i j}^{(\bullet)}$ terms are the unique subcell stress components, the $\mathbf{f}^{m}$ vector and the $f_{i j}^{m}$ terms contain information on the repeating unit cell dimensions and the global (unit cell) strains, the $\mathbf{f}^{I}$ vector and the $f_{i j}^{I(\bullet)}$ terms contain the inelastic effects, and the $\mathbf{f}^{T}$ vector contains the thermal effects.

HFGMC, on the other hand, with its higher-order displacement field (Eq. 3), requires supplementary equations in its formulation. In addition to the imposition of continuity of displacements and tractions between subcells in an integral sense, the zeroth, first, and second moments of the local (subcell) equilibrium equations are satisfied in a volumetric sense. Finally, periodic boundary conditions are imposed at the repeating unit cell boundaries, resulting in a linear system of $15 N_{\beta} N_{\gamma}$ algebraic equations,

$$
\mathbf{K U}=\mathbf{f}+\mathbf{g}
$$

where the $\mathbf{K}$ matrix contains information on the subcell material elastic properties and the subcell dimensions, $\mathbf{U}$ contains the unknown terms $W_{i(\bullet)}^{(\beta \gamma)}$ in the displacement field (Eq. 3), the $\mathbf{f}$ vector contains information on the global (unit cell) strains and the thermal effects, and $\mathbf{g}$ contains integrals of inelastic strains. Clearly, solving Eq. (6) for the $15 N_{\beta} N_{\gamma}$ unknowns in HFGMC is considerably more computationally demanding than solving Eq. (4) for the $N_{\beta}+N_{\gamma}$ unknowns (in addition to the $N_{\beta}+N_{\gamma}+1$ decoupled equations (5)) in GMC.

Once Eqs. $(4-6)$ are solved, the local stress and strain fields throughout the repeating unit cell can be determined from the standard kinematics equations and the local constitutive equations (1) in both GMC and HFGMC. Then the terms in the global constitutive equation,

$$
\overline{\boldsymbol{\sigma}}=\mathbf{C}^{*}\left(\overline{\boldsymbol{\varepsilon}}-\overline{\boldsymbol{\varepsilon}}^{I}-\overline{\boldsymbol{\varepsilon}}^{T}\right)
$$

can be determined using the definition of average (global) stress,

$$
\overline{\boldsymbol{\sigma}}=\frac{1}{h l} \sum_{\beta=1}^{N_{\beta}} \sum_{\gamma=1}^{N_{\gamma}} h_{\beta} l_{\gamma} \boldsymbol{\sigma}^{(\beta \gamma)}
$$


where $\mathbf{C}^{*}$ is the average or effective stiffness matrix, and $\overline{\boldsymbol{\varepsilon}}, \overline{\boldsymbol{\varepsilon}}^{I}$, and $\overline{\boldsymbol{\varepsilon}}^{T}$ are the average or effective total, inelastic, and thermal strain vectors, respectively.

Eq. (7) is the effective (macro) constitutive equation for the homogenized composite material represented by the GMC or HFGMC repeating unit cell. It allows one to impose an admissible combination of global stress and strain components, in addition to spatially constant thermal loading, and to determine the remaining global stresses and strains acting on the composite material. Then, via Eqs. $(4-6)$ the local stress and strain fields can be determined as well. These local-global fields constitute the complete micromechanics solution.

Table 1 provides a comparison of the features of the GMC and HFGMC micromechanics models. As mentioned previously, GMC, with its first-order displacement field (Eq. 2) lacks shear coupling. While this is a limitation in terms of local field accuracy, it is this lack of shear coupling that enabled GMC to be reformulated in order to provide its superior computational efficiency. This limitation has been overcome by HFGMC, which now includes shear coupling, but the associated improved local field accuracy comes at an additional computational cost. Further, GMC's lack of shear coupling is manifested in piece-wise uniform stress and strain fields throughout the repeating unit cell. In contrast, the fields in HFGMC vary within the subcells, and in the presence of inelasticity and damage, this spatial field variation is arbitrary. As a result, HFGMC must employ a number of integration points within each subcell to represent the higher-order local fields, while GMC requires the local field variables to be stored only once for each subcell.

Table 1 Comparison of the GMC and HFGMC micromechanics models.

\begin{tabular}{l|l|l}
\hline \hline & GMC & HFGMC \\
\hline General global accuracy & Very good & Excellent \\
Computational efficiency & Excellent & Fair \\
Local field accuracy & Fair & Excellent \\
Shear coupling & No & Yes \\
Subcell mesh dependence & No & Yes \\
Admits local inelasticity & Yes & Yes \\
Suitable for inclusion in structural models & Yes & Yes \\
Multi-axial & Yes & Yes \\
\hline \hline
\end{tabular}

\section{B. Implementation within Classical Lamination Theory}

Within the MAC/GMC software package [7], classical lamination theory $[5,6]$ has been implemented such that the ply material behavior is modeled using GMC of HFGMC, as shown in Fig. 2. An extension of the software is underway to enable utilization of HFGMC to model the ply-level response. As shown in the figure, the GMC repeating unit cell representing the ply composite material is active at two through-thickness Gauss quadrature points within each ply. The points are utilized to track and integrate the nonlinear effects associated with damage, debonding, and inelasticity, which vary arbitrarily within a ply in the general loading case involving damage.

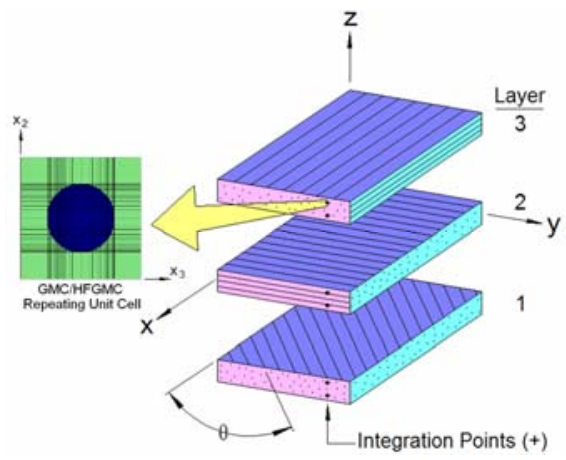

Figure 2. Schematic diagram showing the implementation of GMC or HFGMC within classical lamination theory to model the ply-level behavior at the integration points. 
The laminate constitutive equation can be written as,

$$
\left[\begin{array}{cc}
\overline{\mathbf{A}} & \overline{\mathbf{B}} \\
\overline{\mathbf{B}} & \overline{\mathbf{D}}
\end{array}\right]\left[\begin{array}{l}
\boldsymbol{\varepsilon}^{0} \\
\mathbf{\kappa}
\end{array}\right]=\left[\begin{array}{l}
\mathbf{N} \\
\mathbf{M}
\end{array}\right]+\left[\begin{array}{l}
\mathbf{N}^{T} \\
\mathbf{M}^{T}
\end{array}\right]+\left[\begin{array}{l}
\mathbf{N}^{i n} \\
\mathbf{M}^{i n}
\end{array}\right]
$$

where $\overline{\mathbf{A}}, \overline{\mathbf{B}}$, and $\overline{\mathbf{D}}$ are the effective extension, coupling, and bending stiffness matrices of the laminate, $\boldsymbol{\varepsilon}^{0}$ and $\mathbf{K}$ are vectors containing the midplane strain and curvature components, $\mathbf{N}$ and $\mathbf{M}$ are the force and moment resultant vectors, $\mathbf{N}^{T}$ and $\mathbf{M}^{T}$ are the thermal force and moment vectors, and $\mathbf{N}^{i n}$ and $\mathbf{M}^{\text {in }}$ are the inelastic force and moment vectors. The effective stiffness and coefficients of thermal expansion of each layer, once properly transformed to the global (laminate) coordinate system via standard rotation equations, allow the determination of the $\overline{\mathbf{A}}, \overline{\mathbf{B}}$, and $\overline{\mathbf{D}}$ matrices and the thermal force and moment vectors (for a given temperature change). In the absence of inelasticity and damage, the formulation would then be complete. Given laminate scale applied loading in terms of an appropriate combination of mechanical force and moment resultants and midplane strains and curvatures along with a temperature change, the global response of the laminate can be determined using Eq. (9). However, in the presence of inelasticity or damage (the case considered herein), the model must constantly localize and homogenize as the loading is applied in an incremental fashion. That is, the laminate scale loading results in a stress and strain field at the integration points within each layer. These fields are transformed (via rotation) and passed to GMC, which further localizes the fields from the level of the unit cell to each individual subcell. Then, on this micro scale, the failure criterion is evaluated, and if failure is present, the local elastic properties of the subcell are degraded. GMC then determines a new effective stiffness for the composite material based on the presence of failed subcells, which, once transformed to the laminate coordinate system, is representative of a particular integration point in a particular layer of the laminate. Finally, the stiffness is integrated through the thickness of each layer and the new effective $\overline{\mathbf{A}}, \overline{\mathbf{B}}$, and $\overline{\mathbf{D}}$ matrices are determined via the appropriate lamination theory equations. As stated, this localization/homogenization process must occur at each increment of the applied loading.

\section{Failure Theories}

The failure theories compared herein, all of which are applied on the fiber/matrix constituent level, are the maximum stress, maximum strain, Tsai-Hill, and Tsai-Wu criteria. A comparison of these failure theories applied at the ply level is provided by Herakovich [5]. The maximum stress criterion can be expressed as,

$$
\begin{gathered}
X_{C}<\sigma_{11}<X_{T} \\
Y_{C}<\sigma_{22}<Y_{T} \\
Z_{C}<\sigma_{33}<Z_{T} \\
\left|\tau_{23}\right|<Q \\
\left|\tau_{13}\right|<R \\
\left|\tau_{12}\right|<S
\end{gathered}
$$

where the stress components are subcell values, $X_{T}, Y_{T}$, and $Z_{T}$ are the tensile material normal strengths, $X_{C}, Y_{C}$, and $Z_{C}$ are the compressive material normal strengths, and $Q, R$, and $S$ are the material shear strengths. Note that compressive strengths are negative. Violation of any of Eqs. (10) indicates failure of the subcell.

Similarly, the maximum strain criterion can be written as,

$$
\begin{aligned}
X_{\varepsilon C} & <\varepsilon_{11}<X_{\varepsilon T} \\
Y_{\varepsilon C} & <\varepsilon_{22}<Y_{\varepsilon T} \\
Z_{\varepsilon C} & <\sigma_{33}<Z_{\varepsilon T}
\end{aligned}
$$




$$
\begin{aligned}
& \left|\gamma_{23}\right|<Q_{\varepsilon} \\
& \left|\gamma_{13}\right|<R_{\varepsilon} \\
& \left|\gamma_{12}\right|<S_{\varepsilon}
\end{aligned}
$$

where the strain components are subcell values, $X_{\varepsilon T}, Y_{\varepsilon T}$, and $Z_{\varepsilon T}$ are the tensile material normal failure strains, $X_{\varepsilon C}$, $Y_{\varepsilon C}$, and $Z_{\varepsilon C}$ are the compressive material normal failure strains, and $Q_{\varepsilon}, R_{\varepsilon}$, and $S_{\varepsilon}$ are the material shear failure. Note that compressive failure strains are negative. Violation of any of Eqs. (11) indicates failure of the subcell.

The fully multiaxial Tsai-Hill criterion [18] failure surface can be expressed as,

$$
\begin{aligned}
& \frac{\sigma_{11}^{2}}{X^{2}}+\frac{\sigma_{22}^{2}}{Y^{2}}+\frac{\sigma_{33}^{2}}{Z^{2}}+\frac{\tau_{23}^{2}}{Q^{2}}+\frac{\tau_{13}^{2}}{R^{2}}+\frac{\tau_{12}^{2}}{S^{2}} \\
& -\sigma_{11} \sigma_{22}\left(\frac{1}{X^{2}}+\frac{1}{Y^{2}}-\frac{1}{Z^{2}}\right)-\sigma_{11} \sigma_{33}\left(\frac{1}{X^{2}}-\frac{1}{Y^{2}}+\frac{1}{Z^{2}}\right)-\sigma_{22} \sigma_{33}\left(-\frac{1}{X^{2}}+\frac{1}{Y^{2}}+\frac{1}{Z^{2}}\right)=1
\end{aligned}
$$

where, in order to incorporate differing tensile and compressive strengths,

$$
\begin{aligned}
& X= \begin{cases}X_{T} & \sigma_{11} \geq 0 \\
X_{C} & \sigma_{11}<0\end{cases} \\
& Y= \begin{cases}Y_{T} & \sigma_{22} \geq 0 \\
Y_{C} & \sigma_{22}<0\end{cases} \\
& Z= \begin{cases}Z_{T} & \sigma_{33} \geq 0 \\
Z_{C} & \sigma_{33}<0\end{cases}
\end{aligned}
$$

Subcell failure is indicated when the function on the left hand side of Eq. (12) is greater than 1.

Finally, the Tsai-Wu criterion [19] failure surface can be written as,

$$
\begin{aligned}
& \sigma_{11}\left(\frac{1}{X_{T}}+\frac{1}{X_{C}}\right)+\sigma_{22}\left(\frac{1}{Y_{T}}+\frac{1}{Y_{C}}\right)+\sigma_{33}\left(\frac{1}{Z_{T}}+\frac{1}{Z_{C}}\right)-\frac{\sigma_{11}^{2}}{X_{T} X_{C}}-\frac{\sigma_{22}^{2}}{Y_{T} Y_{C}}-\frac{\sigma_{33}^{2}}{Z_{T} Z_{C}}+\frac{\tau_{23}^{2}}{Q^{2}}+\frac{\tau_{13}^{2}}{R^{2}}+\frac{\tau_{12}^{2}}{S^{2}} \\
& +2 F_{12} \sigma_{11} \sigma_{22}+2 F_{13} \sigma_{11} \sigma_{33}+2 F_{23} \sigma_{22} \sigma_{33}=1
\end{aligned}
$$

where the compressive strengths are negative. The interaction coefficients, $F_{12}, F_{13}$, and $F_{23}$, herein have been taken according to Tsai and Hahn [20],

$$
\begin{aligned}
& F_{12}=-\frac{1}{2 \sqrt{X_{T} X_{C} Y_{T} Y_{C}}} \\
& F_{13}=-\frac{1}{2 \sqrt{X_{T} X_{C} Z_{T} Z_{C}}} \\
& F_{23}=-\frac{1}{2 \sqrt{Y_{T} Y_{C} Z_{T} Z_{C}}}
\end{aligned}
$$

Note that the Tsai-Wu theory with the interaction terms taken according to Ref. 20 have been denoted "Tsai-Wu (Hahn)". Subcell failure is indicated when the function on the left hand side of Eq. (14) is greater than 1. 


\section{Results}

The laminate lay-ups, materials, and loading cases are shown in table 2 and were extracted from Ref. 2.

Table 2 Summary of laminate types, material types and required plots.

\begin{tabular}{lll}
\hline Laminate lay-up & Material & Loading case and description \\
\hline $0^{\circ}$ unidirectional lamina & E-glass/LY556/HT907/DY063 & 1. $\sigma_{\mathrm{y}}$ vs $\tau_{\mathrm{xy}}$ failure stress envelope \\
& T300/BSL914C & 2. $\sigma_{\mathrm{x}}$ vs $\tau_{\mathrm{xy}}$ failure stress envelope \\
& E-glass/MY750/HY917/DY063 & 3. $\sigma_{\mathrm{y}}$ vs $\sigma_{\mathrm{x}}$ failure stress envelope \\
$\left(90^{\circ} / \pm 30^{\circ}\right)_{\mathrm{s}}$ laminate & E-glass/LY556/HT907/DY063 & 4. $\sigma_{\mathrm{y}}$ vs $\sigma_{\mathrm{x}}$ failure stress envelope \\
& & 5. $\sigma_{\mathrm{x}}$ vs $\tau_{\mathrm{xy}}$ failure stress envelope \\
$\left(0^{\circ} / \pm 45^{\circ} / 90^{\circ}\right)_{\mathrm{s}}$ laminate & AS4/3501-6 & 6. $\sigma_{\mathrm{y}}$ vs $\sigma_{\mathrm{x}}$ failure stress envelope \\
& & 7. Stress/strain curve for $\sigma_{\mathrm{y}} / \sigma_{\mathrm{x}}=1 / 0$ \\
& & 8. Stress/strain curve for $\sigma_{\mathrm{y}} / \sigma_{\mathrm{x}}=2 / 1$ \\
$\left( \pm 55^{\circ}\right)_{\mathrm{s}}$ angle ply laminate & E-glass/MY750/HY917/DY063 & 9. $\sigma_{\mathrm{y}}$ vs $\sigma_{\mathrm{x}}$ failure stress envelope \\
& & 10. Stress $/$ strain curve for $\sigma_{\mathrm{y}} / \sigma_{\mathrm{x}}=1 / 0$ \\
& & 11. Stress $/$ strain curve for $\sigma_{\mathrm{y}} / \sigma_{\mathrm{x}}=2 / 1$ \\
$\left(0^{\circ} / 90^{\circ}\right)_{\mathrm{s}}$ cross ply laminate & E-glass/MY750/HY917/DY063 & 12. Stress/strain curve for $\sigma_{\mathrm{y}} / \sigma_{\mathrm{x}}=0 / 1$ \\
$\left( \pm 45^{\circ}\right)_{\mathrm{s}}$ angle ply laminate & E-glass/MY750/HY917/DY063 & 13. Stress/strain curve for $\sigma_{\mathrm{y}} / \sigma_{\mathrm{x}}=1 / 1$ \\
& & 14. Stress/strain curve for $\sigma_{\mathrm{y}} / \sigma_{\mathrm{x}}=1 /-1$ \\
\hline
\end{tabular}

The results shown below are pure predictions based on the fiber/matrix constituent properties provided in Ref. 2 . As such, even the lamina failure envelopes are pure predictions that do not benefit from the "anchor points" on the failure envelope axes associated with the ply failure stresses. In contrast, backing out in-situ constituent properties in order to match the aforementioned anchor points would provide better correlation with the experiment data, but also would bias the predictions. Furthermore, the simplest damage progression model, a step function, has been employed herein. That is, once a subcell has reached failure according to the applicable failure criterion, its stiffness is instantaneous reduced to $0.01 \%$ of its original value. This is done identically, regardless of the mode of failure, and in the case of HFGMC, where stresses vary within a subcell, the subcell average stress is employed to predict failure. Clearly, a much more involved failure progression model can be incorporated in the future, and within HFGMC, subcell quadrature point based failure (rather than subcell average failure) could be considered. In all cases, the $26 \times 26$ subcell repeating unit cell, shown in Fig. 2, was employed.

\section{A. $0^{\circ}$ lamina}

For the following $0^{\circ}$ unidirectional lamina the experimental values are shown as open circles while the four different subcell failure criteria are shown with various line types. The initial failure envelope was not included because the initial failure envelope was only 1 to $2 \mathrm{MPa}$ from the predicted final failure envelope for all the $0^{\circ}$ unidirectional lamina.

\section{E-glass/LY556/HT907/DY063: $\sigma_{y}$ vs $\tau_{x y}$ failure stress envelope}

A comparison of theoretical predictions to experimental results for this load case is shown in Fig. 3 for GMC and Fig. 4 for HFGMC. The best fit for the experimental data was the Tsai-Wu (Hahn) failure criteria. It correlated very well in the transverse tension and compression loading. The pure shear loading was under predicted by around $18 \mathrm{MPa}$ for all failure criteria. This pure shear loading was improved on by HFGMC to a $13 \mathrm{MPa}$ under prediction. There also seems to be some interaction with the shear stress and transverse compressive stress in the experiments that prolongs failure which the model is not capturing at this time. The correlation would clearly benefit from using 
in-situ constituent properties, which would allow the model to reproduce the pure shear loading data point. The differences between the GMC and HFGMC models are small compared to those among the various failure criteria. The Tsai-Hill envelope shows the greatest deviation between GMC and HFGMC.

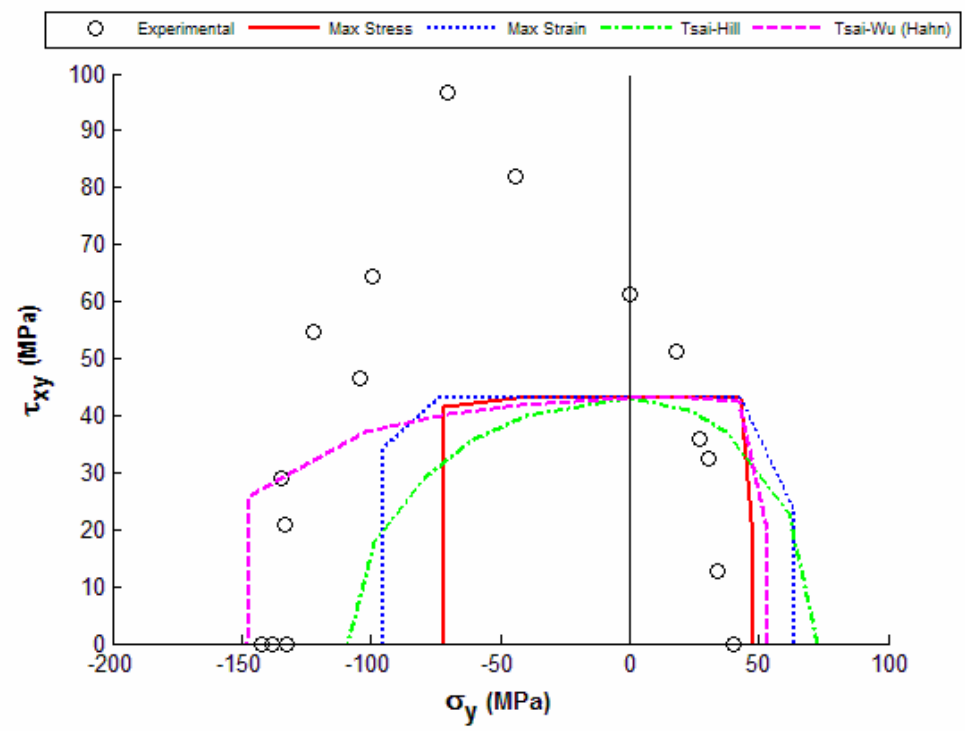

Figure 3. $0^{\circ}$ lamina E-glass/LY556/HT907/DY063: $\sigma_{\mathrm{y}}$ vs $\tau_{\mathrm{xy}}$ failure stress envelope with GMC

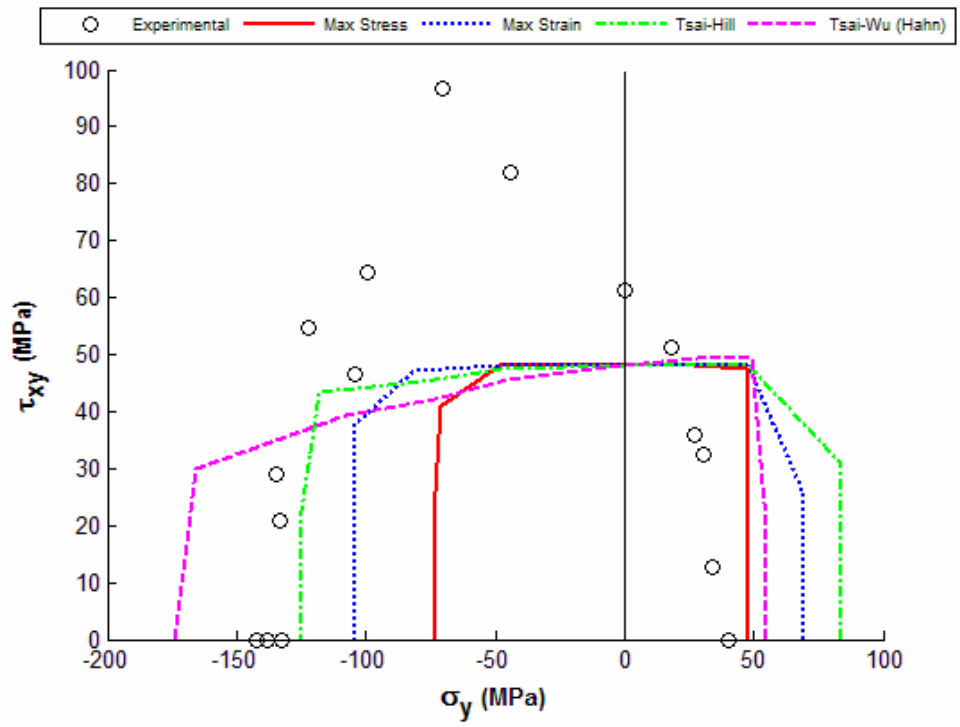

Figure 4. $0^{\circ}$ lamina E-glass/LY556/HT907/DY063: $\sigma_{\mathrm{y}}$ vs $\tau_{\mathrm{xy}}$ failure stress envelope with HFGMC

\section{T300/BSL914C: $\sigma_{x} v s \tau_{x y}$ failure stress envelope}

A comparison of theoretical predictions to experimental results for this load case is shown in Fig. 5 for GMC and Fig. 6 for HFGMC. The various failure criteria are very similar to one another except for the Tsai-Hill and Tsai-Wu (Hahn) having a slightly lower axial tension/shear failure. The difference between the predicted failure shear stress of all the failure criteria and the median failure of the experiments is $20 \mathrm{MPa}$ for GMC and 19 MPa HFGMC. There is a large scatter between the high and low of the experimental data for the shear stress alone with the high being 101.3 MPa and low being 55.2 MPa. This makes it difficult to give an accurate deviation between the experimental 
and predicted data for pure shear loading, although the models are clearly under predicting the shear failure. The deviation between GMC and HFGMC is small.

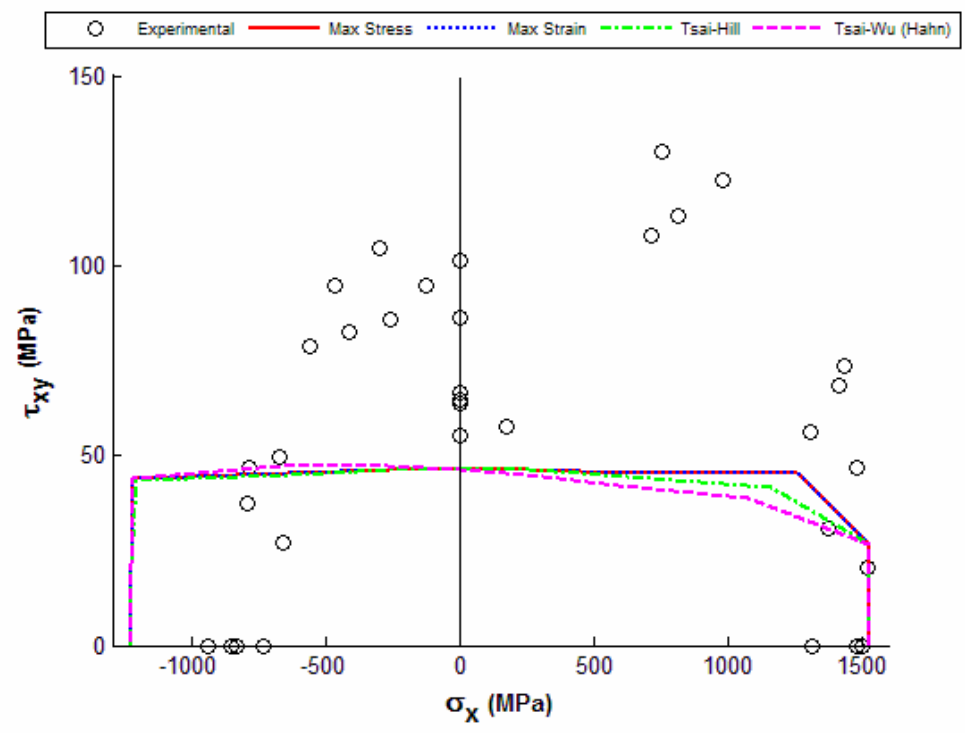

Figure 5. $0^{\circ}$ lamina T300/BSL914C: $\sigma_{\mathrm{x}}$ vs $\tau_{\mathrm{xy}}$ failure stress envelope with GMC

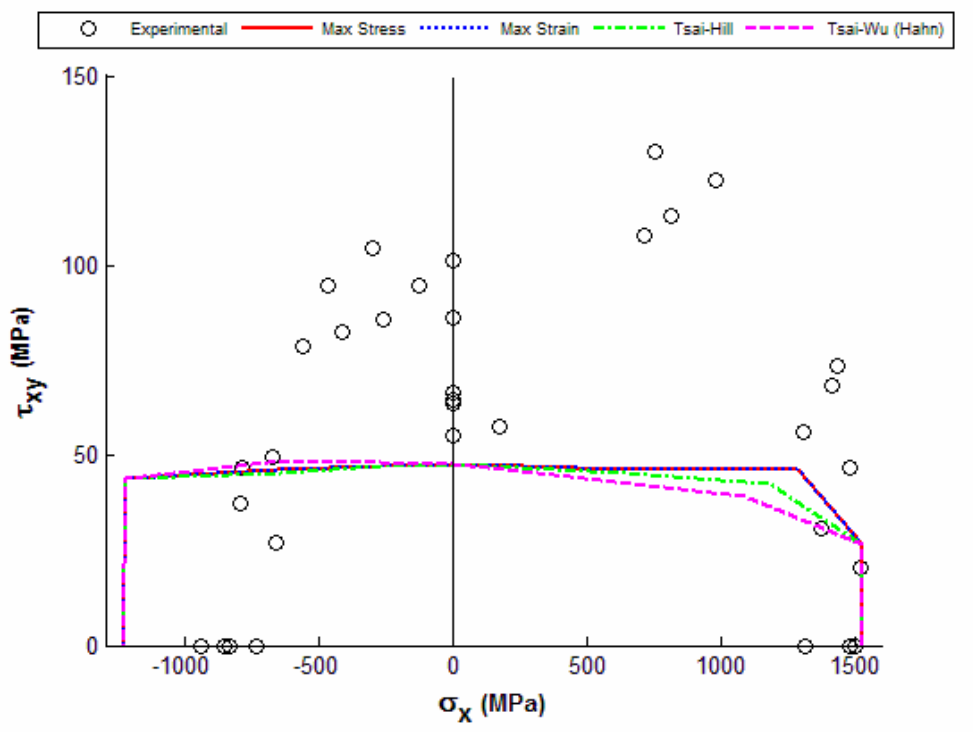

Figure 6. $0^{\circ}$ lamina T300/BSL914C: $\sigma_{\mathrm{x}}$ vs $\tau_{\mathrm{xy}}$ failure stress envelope with HFGMC

\section{E-glass/MY750/HY917/DY063: $\sigma_{y}$ vs $\sigma_{x}$ failure stress envelope}

A comparison of theoretical predictions to experimental results for this load case is shown in Fig. 7 for GMC and Fig. 8 for HFGMC. For the limited experimental data given, one failure criteria fits the data the best. The Tsai-Wu (Hahn) predicts the transverse compressive stress very well and follows the data well in the fourth quadrant for GMC. For the HFGMC, though, Tsai-Wu (Hahn) over predicts the transverse compressive stress, but still predicts the transverse tensile stress well. More test data will be needed to assess which failure criteria are the best at predicting this failure envelope but so far the Tsai-Wu (Hahn) shows good agreement. 


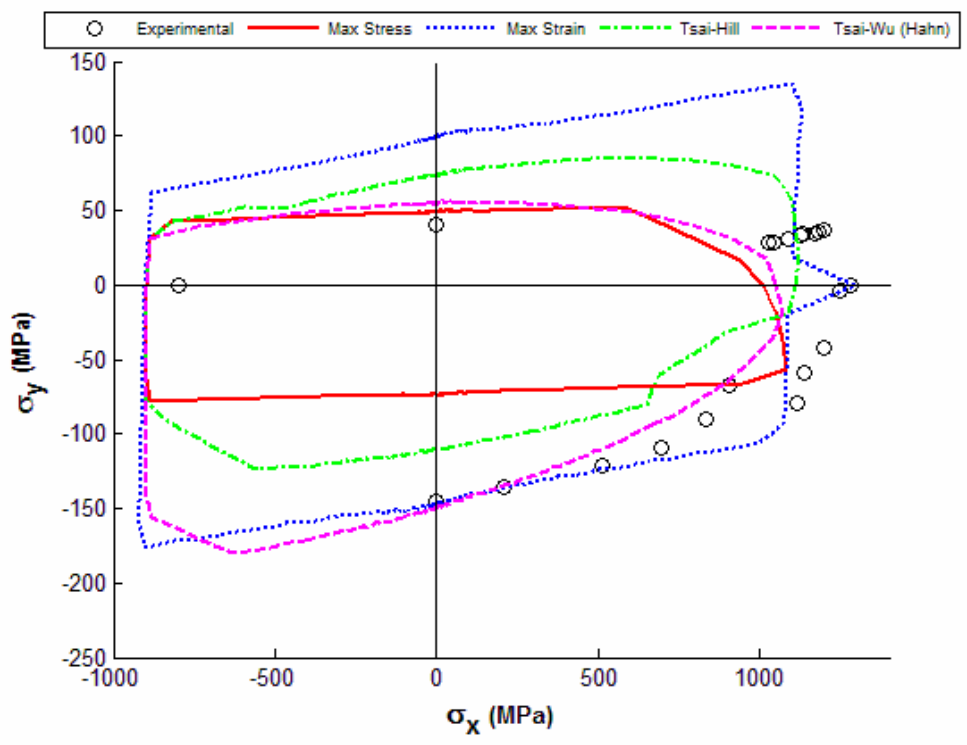

Figure 7. $0^{\circ}$ lamina E-glass/MY750/HY917/DY063: $\sigma_{\mathrm{y}}$ vs $\sigma_{\mathrm{x}}$ failure stress envelope with GMC

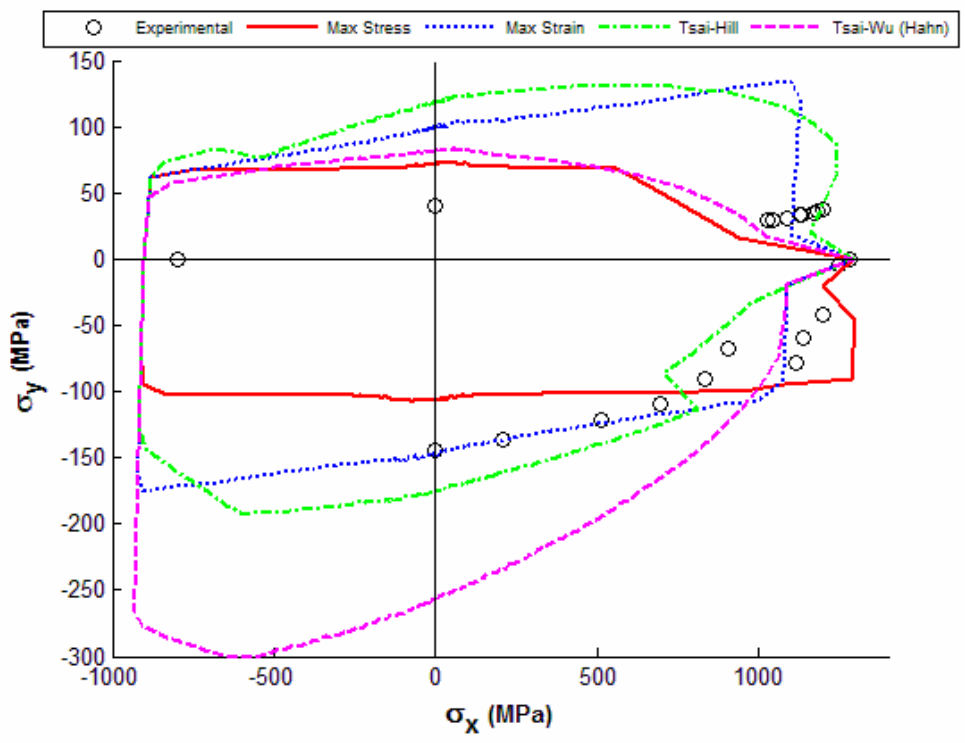

Figure 8. $0^{\circ}$ lamina E-glass/MY750/HY917/DY063: $\sigma_{\mathrm{y}}$ vs $\sigma_{\mathrm{x}}$ failure stress envelope with HFGMC

\section{B. $\left(90^{\circ} / \pm 30^{\circ} / 90^{\circ}\right)$ laminate}

The following $\left(90^{\circ} / \pm 30^{\circ} / 90^{\circ}\right)$ laminate experimental values are shown as open circles while the four different subcell failure criteria are shown with various line types. For this laminate, a significant amount of damage progression was present in the simulations between damage initiation (first subcell failure) and ultimate failure. As such, initial failure envelopes are plotted in addition to the final failure envelopes. In each case, the predicted initial failure envelope is of the same type as its associated failure line type except it has a tic mark added to the line.

\section{E-glass/LY556/HT907/DY063: $\sigma_{y}$ vs $\sigma_{x}$ failure stress envelope}

A comparison of theoretical predictions to experimental results for this load case is shown in Fig. 9 for GMC and in Fig. 10 for HFGMC. All the failure theories seemed to be consistent with one another with minor deviation between them for both the GMC and HFGMC final failure envelopes. They did have varying initial failure 
envelopes with significant differences in the third quadrant (although little experimental data is given in this quadrant). This is also where the failure prediction has the greatest deviation from the experimental data. There is a slight deviation in the second quadrant as well but not as severe in the third quadrant. The first and fourth quadrants agreed quite well with the experimental data. The predictions of GMC and HFGMC are quite similar in this case.

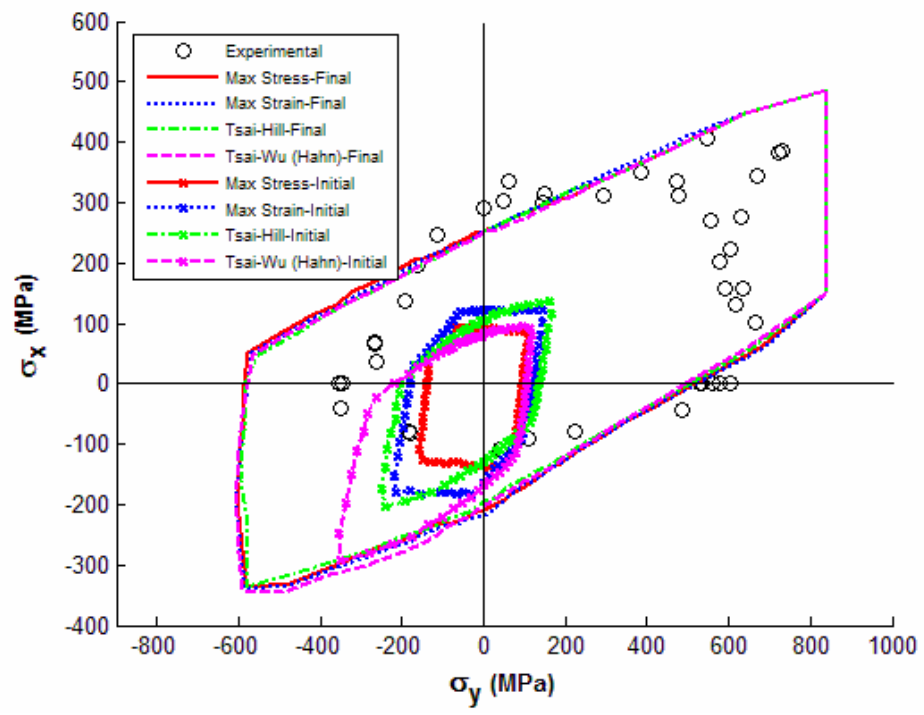

Figure 9. $\left(90^{\circ} / \pm 30^{\circ} / 90^{\circ}\right)$ laminate E-glass/LY556/HT907/DY063: $\sigma_{\mathrm{y}}$ vs $\sigma_{\mathrm{x}}$ failure stress envelope with GMC

- Experimental — Max Stress-Final ……... Max Strain-Final --.--.- Tsai-Hill-Final ----- Tsai-Wu (Hahn)-Final

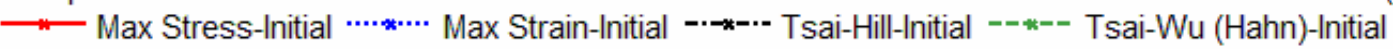

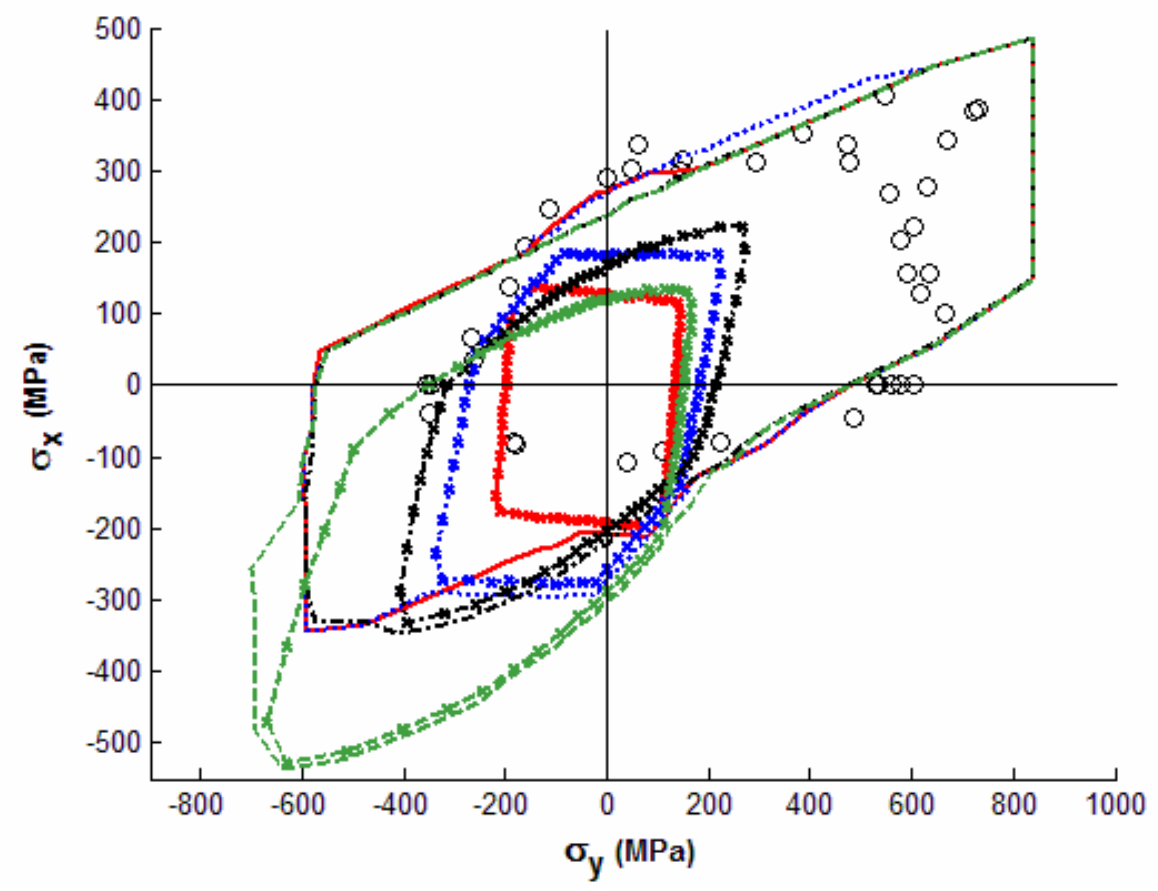

Figure 10. $\left(90^{\circ} / \pm 30^{\circ} / 90^{\circ}\right)$ laminate E-glass/LY556/HT907/DY063: $\sigma_{y}$ vs $\sigma_{x}$ failure stress envelope with HFGMC 


\section{E-glass/LY556/HT907/DY063: $\sigma_{y}$ vs $\tau_{x y}$ failure stress envelope}

A comparison of theoretical predictions to experimental results for this load case is shown in Fig. 11 for GMC and Fig. 12 for HFGMC. The final failure envelopes are very similar to one another, except with the Max Strain failure criterion having a higher prediction for the pure shear stress failure for GMC. The failure criteria have an over prediction for both pure shear and axial compression. The axial tension is a slight under prediction. The initial failure envelopes seemed quite similar with slight variations between them. The initial failure envelopes for the HFGMC tend to come at a higher stresses than those in GMC. Overall, the predictions for this laminate have the general shape of the experimental failure envelope.

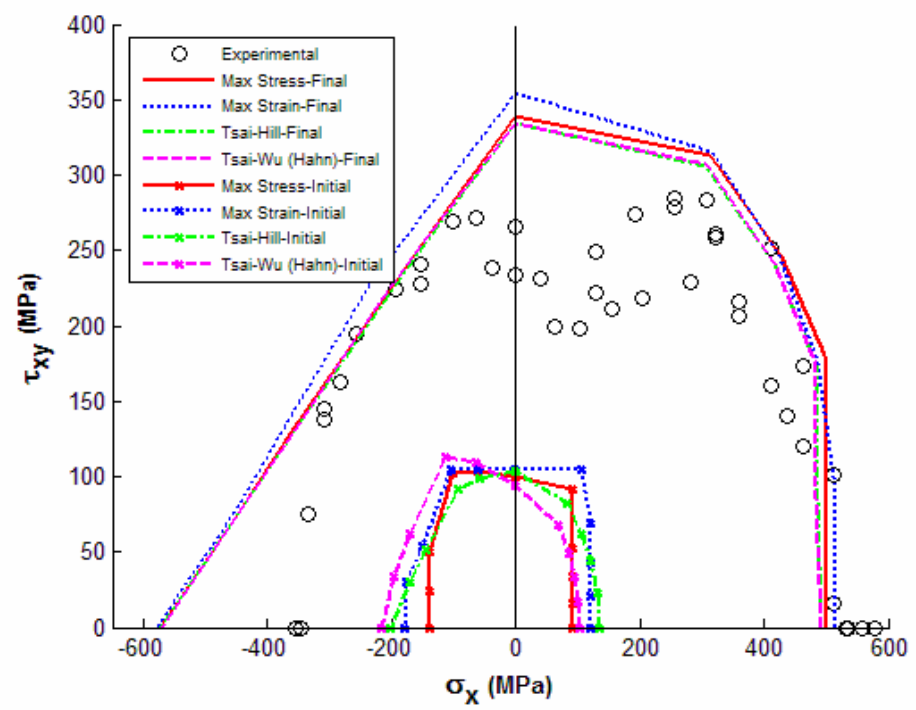

Figure 11. $\left(90^{\circ} / \pm 3^{\circ} / 90^{\circ}\right)$ laminate E-glass/LY556/HT907/DY063: $\sigma_{\mathrm{y}}$ vs $\tau_{\mathrm{xy}}$ failure stress envelope with GMC

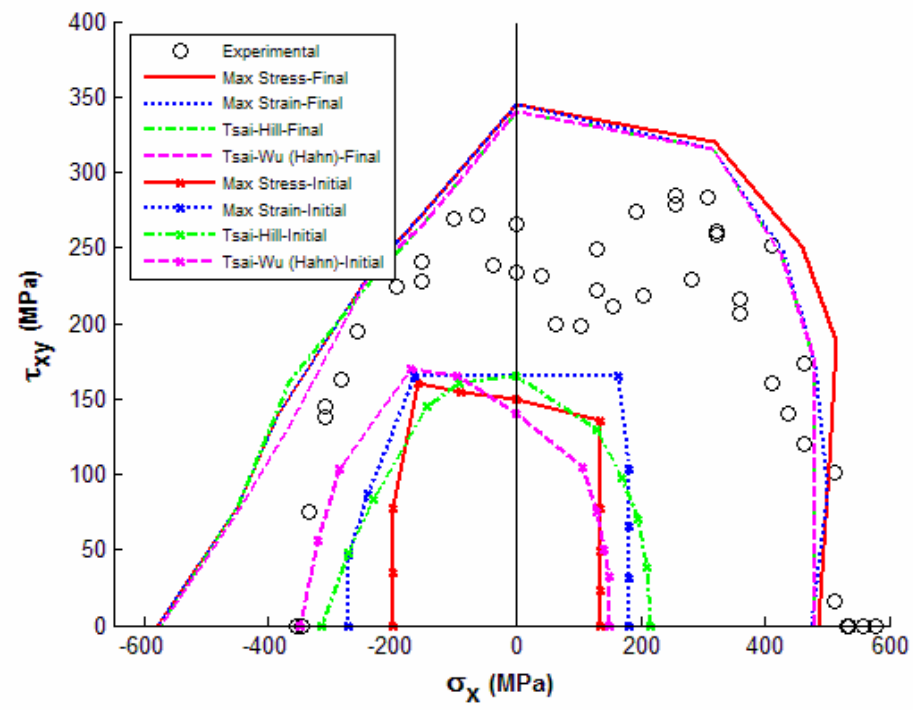

Figure 12. $\left(90^{\circ} / \pm 30^{\circ} / 90^{\circ}\right)$ laminate E-glass/LY556/HT907/DY063: $\sigma_{\mathrm{y}}$ vs $\tau_{\mathrm{xy}}$ failure stress envelope with HFGMC

C. $\left(0^{\circ} / \pm 45^{\circ} / 90^{\circ}\right)$ laminate 


\section{AS4/3501-6: $\sigma_{y}$ vs $\sigma_{x}$ failure stress envelope}

A comparison of theoretical predictions to experimental results for this load case is shown in Fig. 13 for GMC and Fig. 14 for HFGMC. The experimental values are shown as open circles while the four different subcell failure criteria are shown with various line types. The initial failure envelope is of the same type as its associated failure line type except it has a tic mark added to the line.

GMC and HFGMC are similar for all the failure theories, with HFGMC predicting somewhat wider failure initiation envelopes. The final failure envelopes are similar for all the failure criteria for the first, second, and fourth quadrants. The Max Strain and Tsai-Wu (Hahn) have a higher failure point along $225^{\circ}$ than the Max Strain and Tsai-Hill criteria. The failure criteria show good agreement in the first and fourth quadrants while the second has no data. There is an over prediction for all criteria in the third quadrant, however it has been suggested that this third quadrant (compression) data is influenced by specimen buckling. In the initial failure envelope all the failure criteria are similar for the second and fourth quadrant but vary in the first and third.

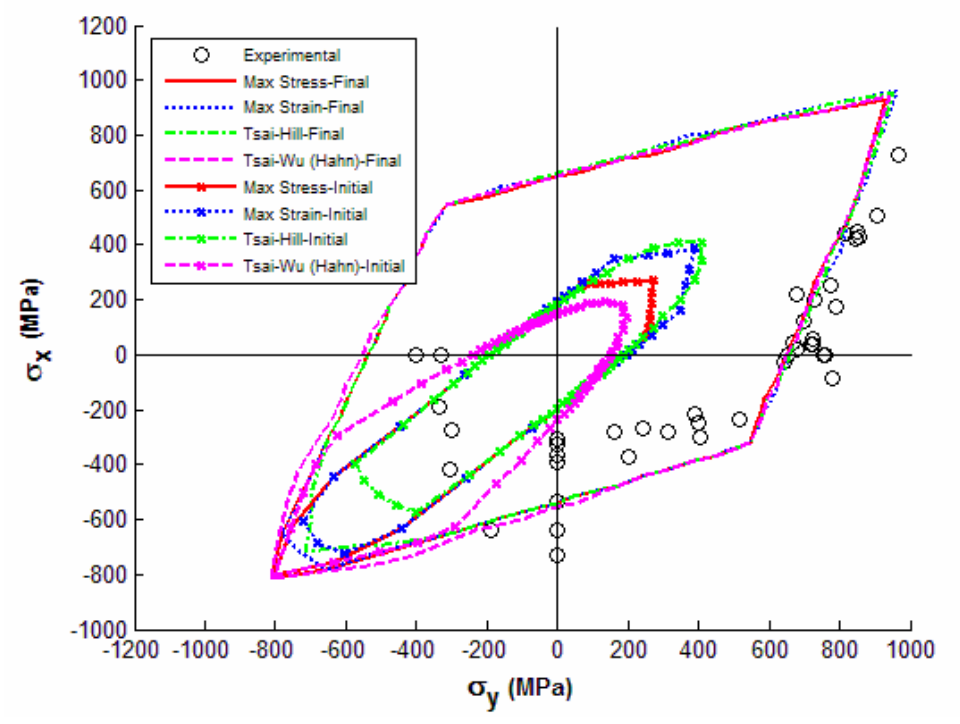

Figure 13. $\left(0^{\circ} / 45^{\circ} / 90^{\circ}\right)$ laminate AS4/3501-6: $\sigma_{\mathrm{y}}$ vs $\sigma_{\mathrm{x}}$ failure stress envelope with GMC

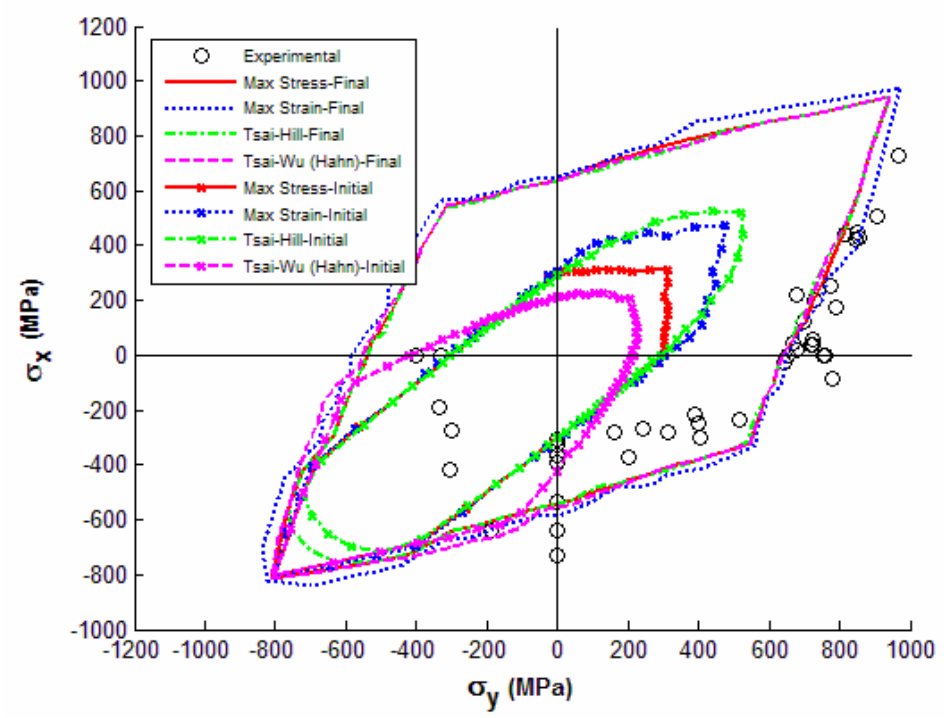

Figure 14. $\left(0^{\circ} / 45^{\circ} / 90^{\circ}\right)$ laminate AS4/3501-6: $\sigma_{y}$ vs $\sigma_{x}$ failure stress envelope with HFGMC 


\section{AS4/3501-6: Stress/strain curves for $\sigma_{y} / \sigma_{x}=1 / 0$}

A comparison of theoretical predictions to experimental results for this load case is shown in Fig. 15 for GMC and Fig. 16 for HFGMC. The experimental values are shown as open circles while the four different subcell failure criteria are shown with various line types.

All of the failure criteria predictions are similar to one another, as are the predictions of GMC and HFGMC. The correlation for the initiation of damage (as indicated by deviation from linearity) between the experiments and the predictions is very good as well. The predictions exhibit a lower slope and the predicted failure is lower than the experimental. This is similar to the results from Bogetti et al. [21] for their ply level theory for this load case.

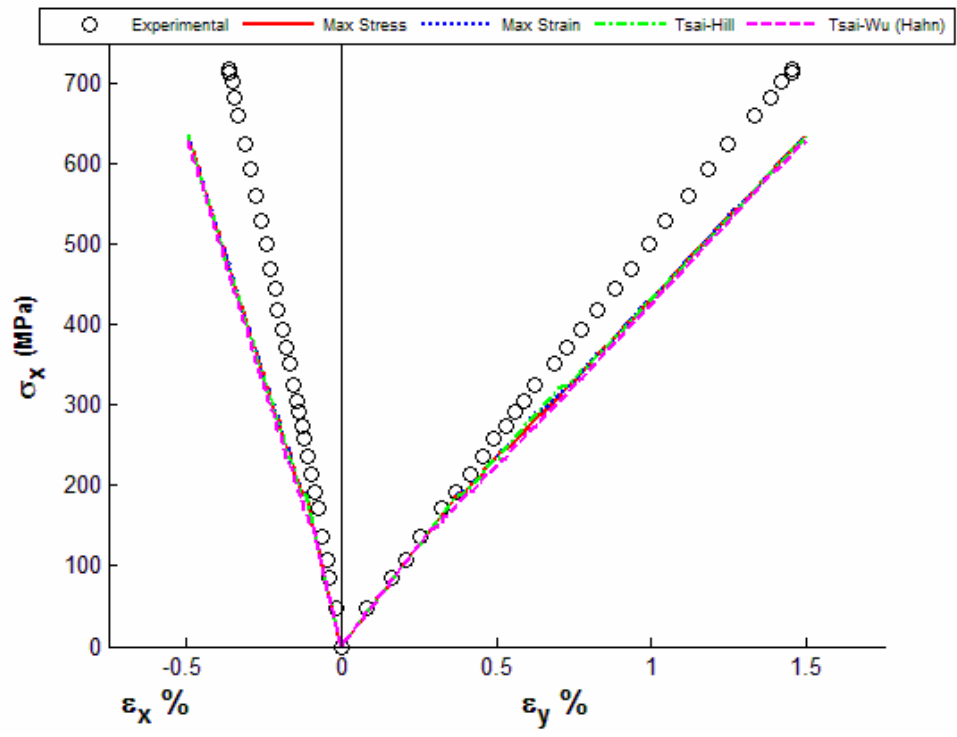

Figure 15. $\left(0^{\circ} / \pm 45^{\circ} / 90^{\circ}\right)$ laminate AS4/3501-6: Stress/strain curves for $\sigma_{y} / \sigma_{x}=0 / 1$ with GMC

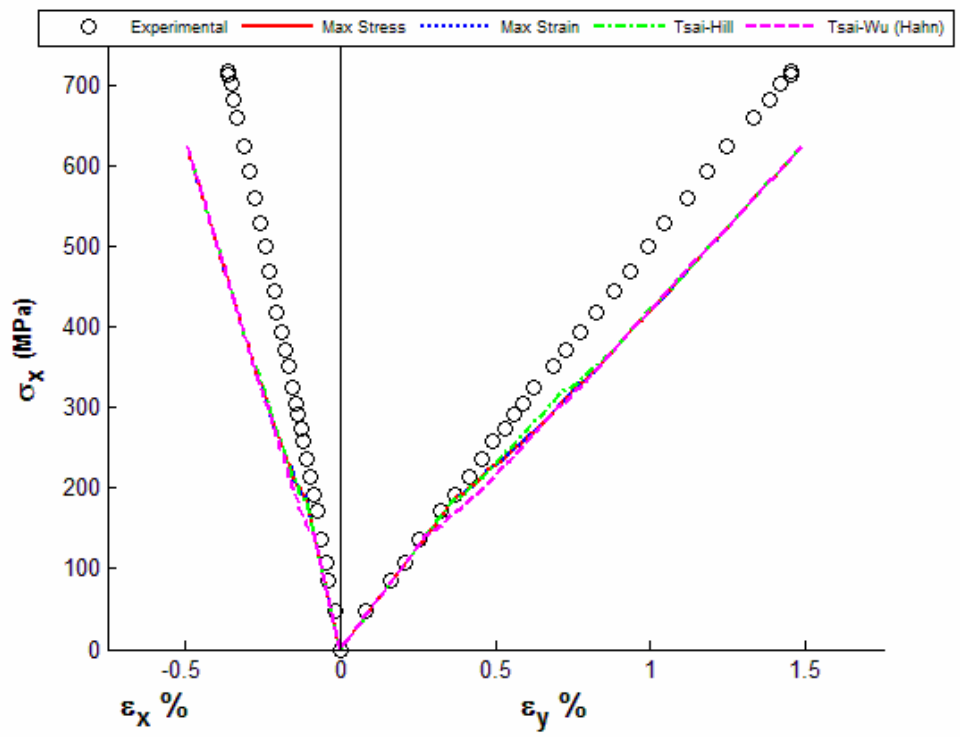

Figure 16. $\left(0^{\circ} / 45^{\circ} / 90^{\circ}\right)$ laminate $\mathrm{AS4} / 3501-6$ : Stress/strain curves for $\sigma_{\mathrm{y}} / \sigma_{\mathrm{x}}=0 / 1$ with HFGMC

8. AS4/3501-6: Stress/strain curves for $\sigma_{y} / \sigma_{x}=2 / 1$

A comparison of theoretical predictions to experimental results for this load case is shown in Fig. 17 for GMC and Fig. 18 for HFGMC. The experimental values are shown as open circles while the four different subcell failure criteria are shown with various line types. 
The various failure criteria predictions are similar to one another. For the GMC case, the failure criteria all under predict the final failure and over predict the nonlinearity caused by progressive failure, but the correlation is better than in the case of $\sigma_{\mathrm{y}} / \sigma_{\mathrm{x}}=0 / 1$. Both Max Stress and Max Strain failure criteria follow the experimental data in the axial direction slightly better than the Tsai-Hill and Tsai-Wu (Hahn) criteria. For the HFGMC case it is similar to the GMC case but the Tsai-Hill has slightly better agreement with the experiment.

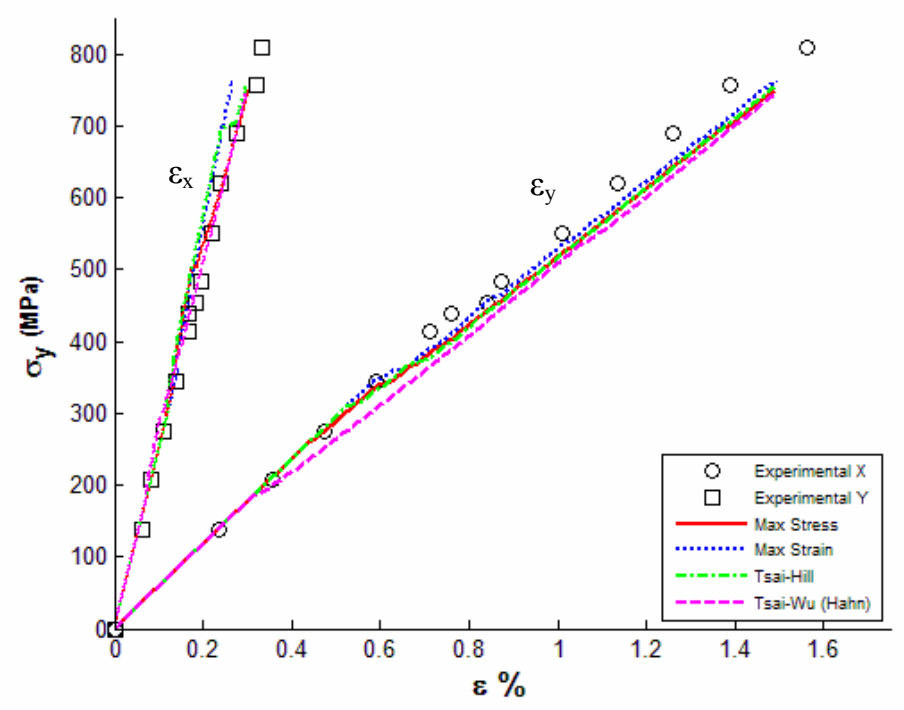

Figure 17. $\left(0^{\circ} / \pm 45^{\circ} / 90^{\circ}\right)$ laminate AS4/3501-6: Stress/strain curve for $\sigma_{\mathrm{y}} / \sigma_{\mathrm{x}}=2 / 1$ with GMC

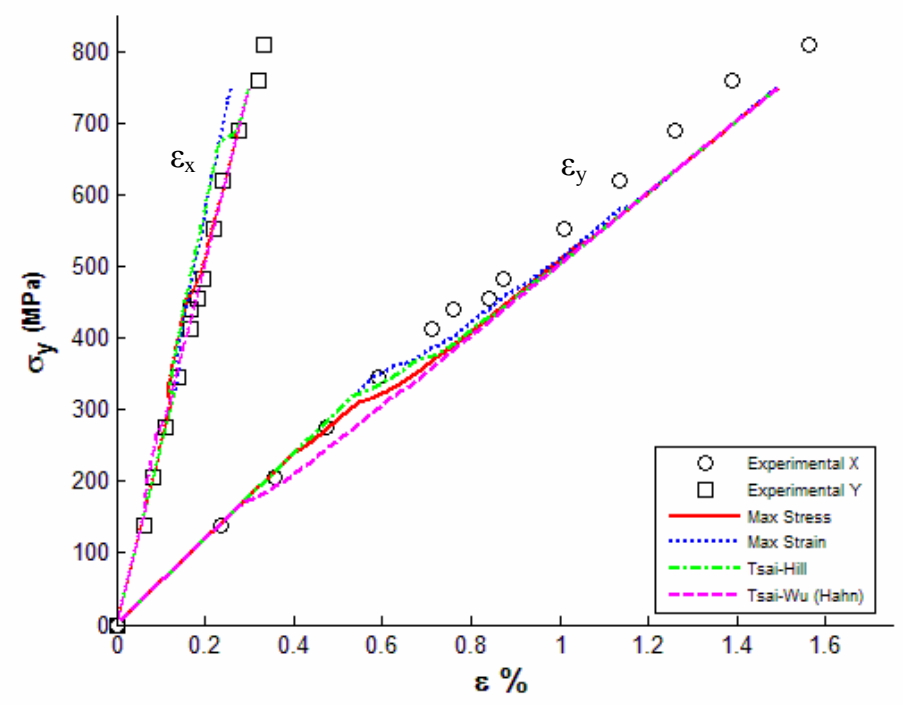

Figure 18. $\left(0^{\circ} / \pm 5^{\circ} / 90^{\circ}\right)$ laminate AS4/3501-6: Stress/strain curve for $\sigma_{\mathrm{y}} / \sigma_{\mathrm{x}}=2 / 1$ with HFGMC

\section{D. $\pm 55^{\circ}$ angle ply laminate}

\section{E-glass/MY750/HY917/DY063: $\sigma_{y}$ vs $\sigma_{x}$ failure stress envelope}

A comparison of theoretical predictions to experimental results for this load case is shown in Figure 19 for GMC and Fig. 20 for HFGMC. The experimental values are shown as open circles while the four different subcell failure criteria are shown with various line types. The initial failure envelope was not included because the initial envelope was only 1 to $2 \mathrm{MPa}$ from the predicted final failure envelope for all failure criteria. 
The simulated failure envelopes do not predict the experimental data very well for GMC. The HFGMC captures the data set better, predicting larger failure envelopes, especially in the third quadrant. The Tsai-Wu (Hahn) criterion seems to provide the best prediction in the case of HFGMC.

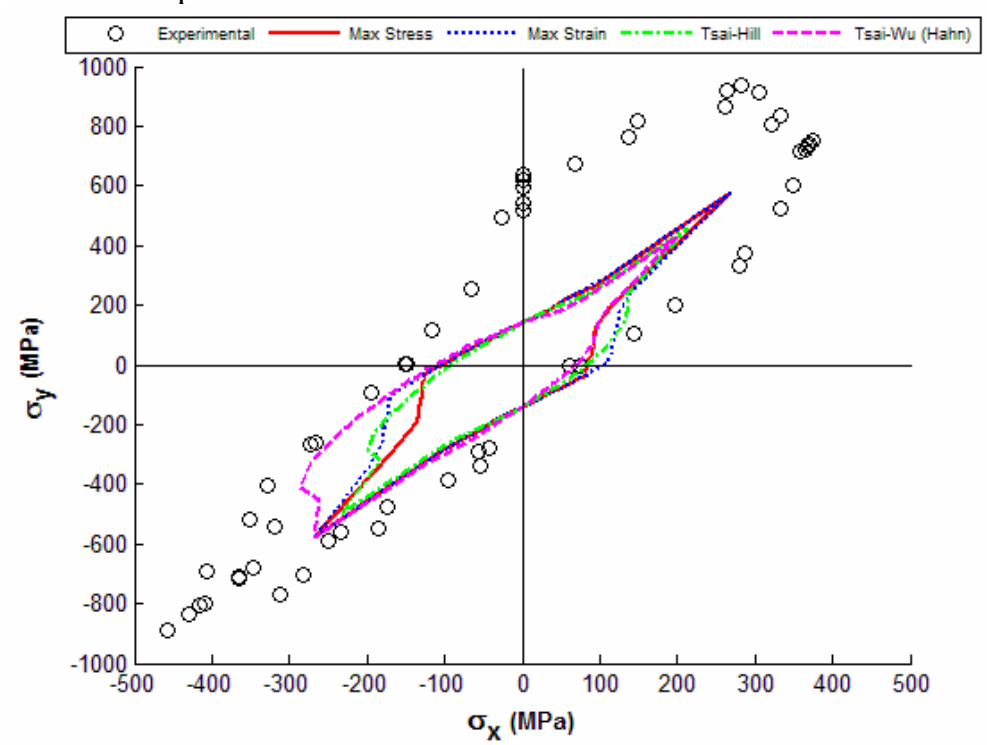

Figure 19. $\pm 55^{\circ}$ angle ply laminate E-glass/MY750/HY917/DY063: $\sigma_{y}$ vs $\sigma_{x}$ failure stress envelope with GMC

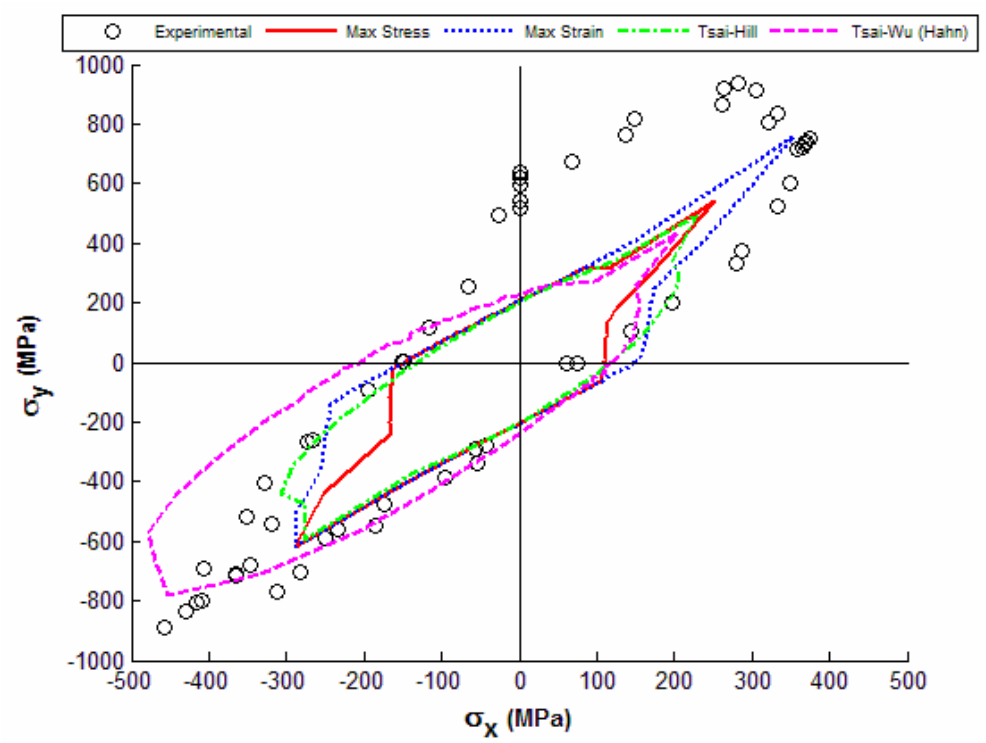

Figure 20. $\pm 55^{\circ}$ angle ply laminate E-glass/MY750/HY917/DY063: $\sigma_{\mathrm{y}}$ vs $\sigma_{\mathrm{x}}$ failure stress envelope with HFGMC

10. E-glass/MY750/HY917/DY063: Stress/strain curves for $\sigma_{y} / \sigma_{x}=1 / 0$

A comparison of theoretical predictions to experimental results for this load case is shown in Fig. 21 for GMC and Fig. 22 for HFGMC. The experimental values are shown as open circles while the four different subcell failure criteria are shown with various line types.

The predictions of the various failure criteria are very similar to one another. They all under predict the final failure significantly, with very little damage progression. This significant under prediction is evident in Figs. 19 and 21 along the positive $\sigma_{\mathrm{y}}$ axis. Neither GMC nor HFGMC correlate well with the experiments. Clearly a mechanism is not being captured by the models as the observed failure response is much more gradual and progressive than the model predictions. It is noted that the present MAC/GMC simulations are based on plate geometry for the laminate, 
while the experimental specimens were tubular. Bogetti et al. [21]suggest that models for this WWFE laminate need to adapt to account for the fiber realignment in the tubes and also to radial expansion or contraction of the tube. This holds true for the $\pm 55^{\circ}$ and $\pm 45^{\circ}$ test specimens whose ply level strain state is dominated by shear. An improved matrix level damage progression model (beyond the step function used herein) could also improve the correlation for this case.

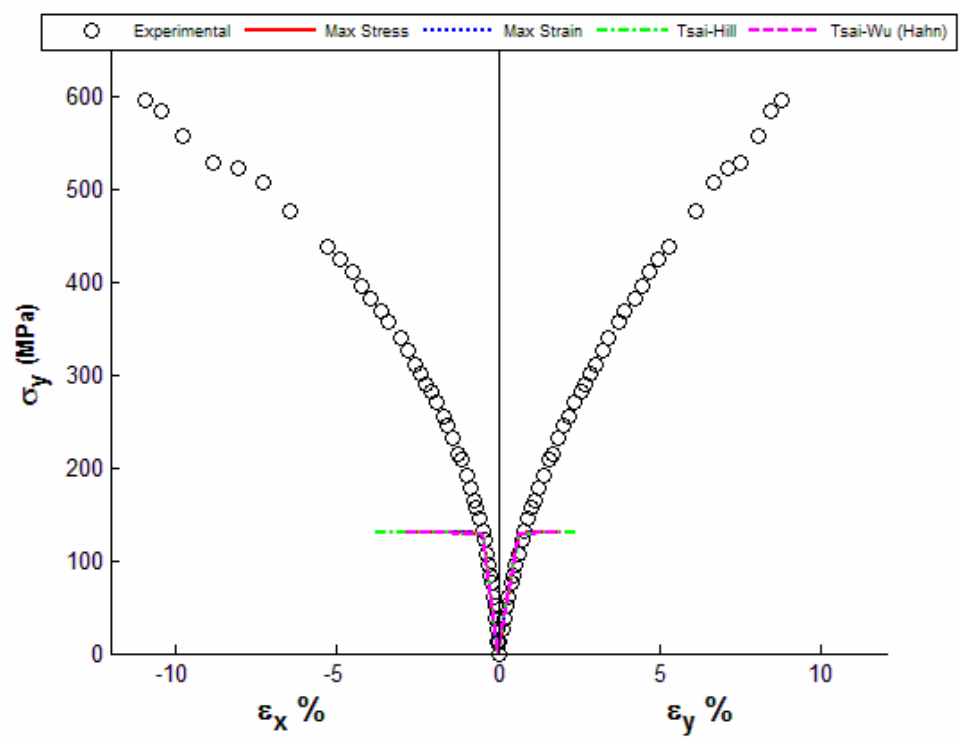

Figure $21 . \pm 55^{\circ}$ angle ply laminate E-glass/MY750/HY917/DY063: Stress/strain curves for $\sigma_{\mathrm{y}} / \sigma_{\mathrm{x}}=1 / 0$ with GMC

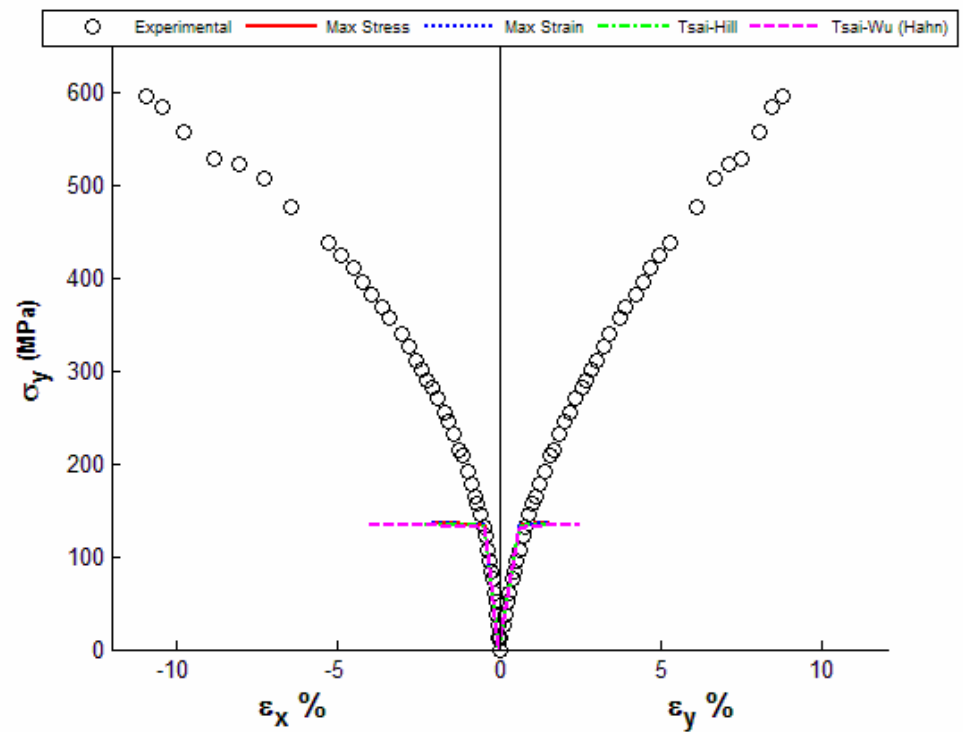

Figure $22 . \pm 55^{\circ}$ angle ply laminate E-glass/MY750/HY917/DY063: Stress/strain curves for $\sigma_{\mathrm{y}} / \sigma_{\mathrm{x}}=1 / 0$ with HFGMC

11. E-glass/MY750/HY917/DY063: Stress/strain curves for $\sigma_{y} / \sigma_{x}=2 / 1$

A comparison of theoretical predictions to experimental results for this load case is shown in Figure 23 for GMC and Fig. 24 for HFGMC. The experimental values are shown as open circles and squares while the four different subcell failure criteria predictions are shown with various line types. 
For the GMC there is a large deviation between the different failure theory predictions. All of the theories have the same shape and capture the experimental data reasonably well. The max strain theory best captures the final failure. As for the HFGMC it did not capture the failure well in this load case. It under predicted final failure for all failure criteria, but followed the shape of the experimental curve well up until its predicted final failure.

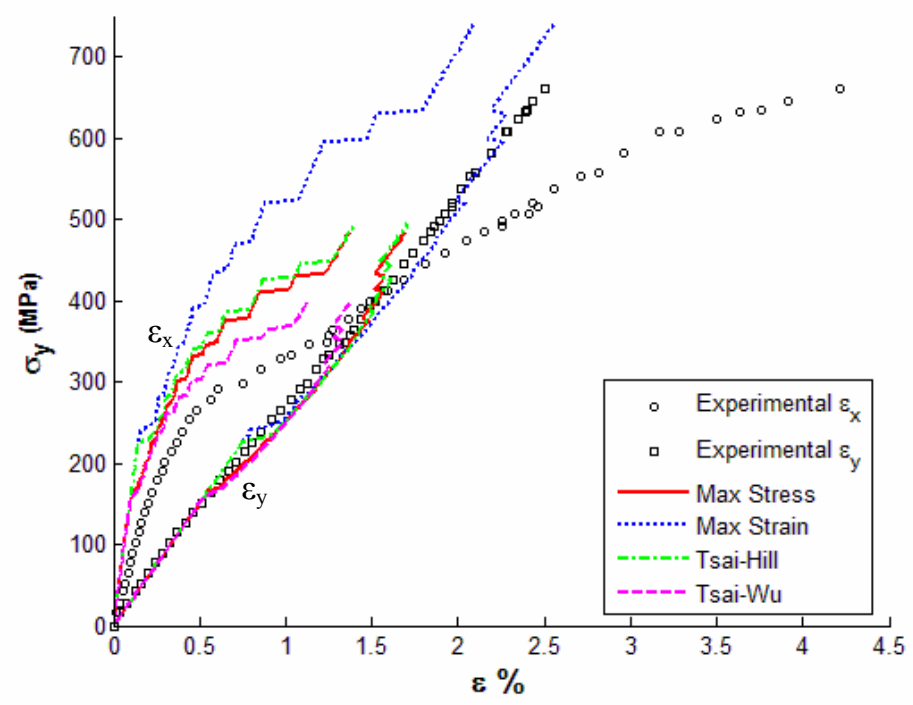

Figure 23. $\pm 55^{\circ}$ angle ply laminate E-glass/MY750/HY917/DY063: Stress/strain curve for $\sigma_{\mathrm{y}} / \sigma_{\mathrm{x}}=2 / 1$ with GMC

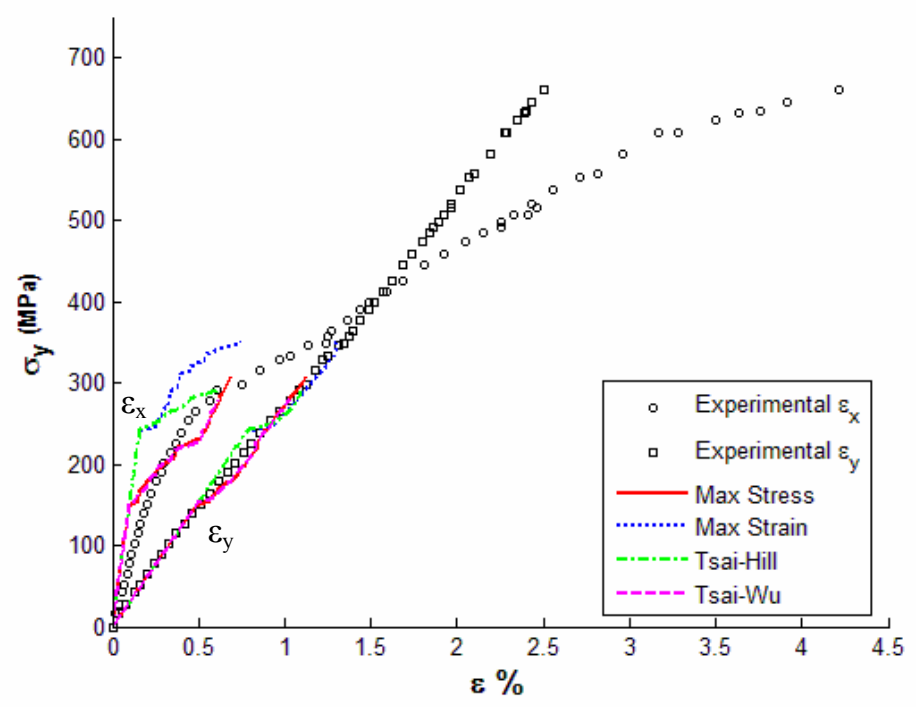

Figure 24. $\pm 55^{\circ}$ angle ply laminate E-glass/MY750/HY917/DY063: Stress/strain curve for $\sigma_{\mathrm{y}} / \sigma_{\mathrm{x}}=2 / 1$ with HFGMC

\section{E. $\left(0^{\circ} / 90^{\circ}\right)$ cross ply laminate}

\section{E-glass/MY750/HY917/DY063: Stress/strain curve for $\sigma_{y} / \sigma_{x}=0 / 1$}

A comparison of theoretical predictions to experimental results for this load case is shown in Fig. 25 for GMC and Fig. 26 for HFGMC. The experimental values are shown as open circles while the four different subcell failure criteria are shown with various line types. 
For both the GMC and HFGMC the various failure criteria predictions are very similar to one another. They all over predict the final failure by $40 \mathrm{MPa}$. For the GMC the failure theories all follow the $\varepsilon_{\mathrm{y}}$ strain well but the Max Strain and Tsia-Hill failure criteria follow the $\varepsilon_{\mathrm{x}}$ strain curve better than the other two failure criteria. For the HFGMC the failure theories all follow the $\varepsilon_{y}$ strain curve well with the Max Strain criterion curve now matching the other criteria curves closely.

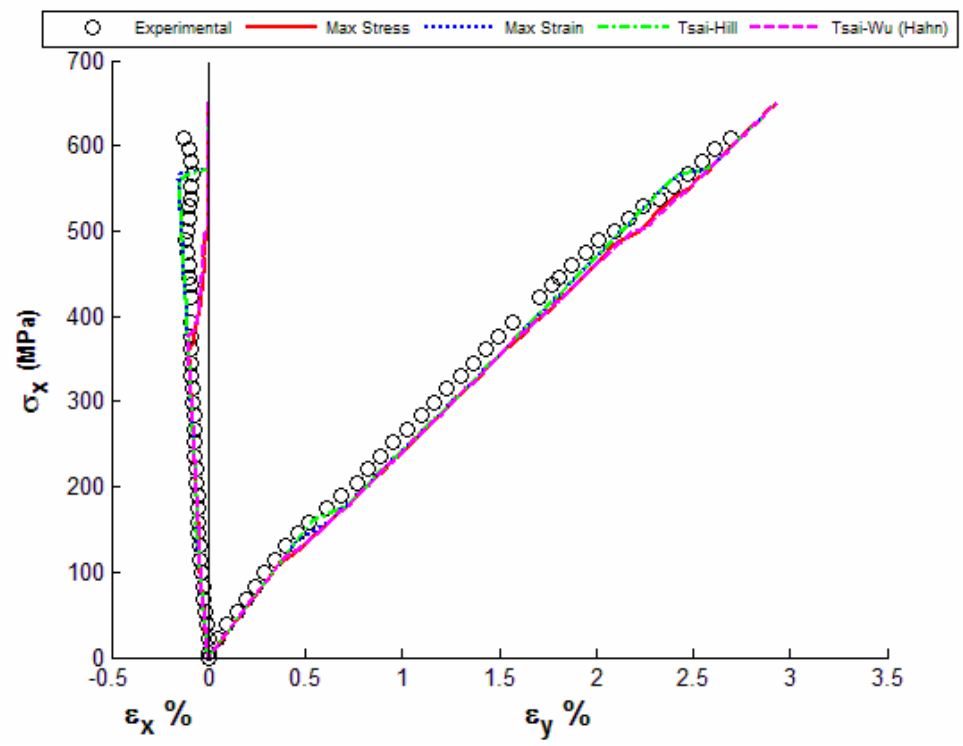

Figure 25. $\left(0^{\circ} / 90^{\circ}\right)$ cross ply laminate E-glass/MY750/HY917/DY063: Stress/strain curve for $\sigma_{\mathrm{y}} / \sigma_{\mathrm{x}}=0 / 1$ with GMC

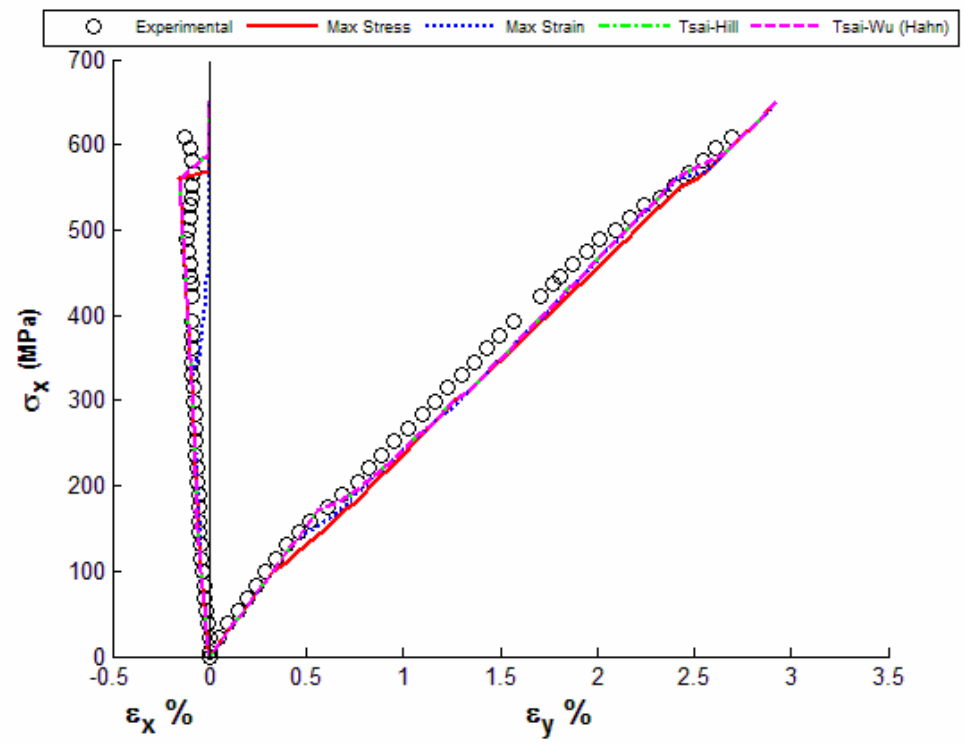

Figure 26. $\left(0^{\circ} / 90^{\circ}\right)$ cross ply laminate E-glass/MY750/HY917/DY063: Stress/strain curve for $\sigma_{\mathrm{y}} / \sigma_{\mathrm{x}}=0 / 1$ with HFGMC

\section{F. $\pm 45^{\circ}$ angle ply laminate}

\section{E-glass/MY750/HY917/DY063: Stress/strain curve for $\sigma_{y} / \sigma_{x}=1 / 1$}

A comparison of theoretical predictions to experimental results for this load case is shown in Fig. 27 for GMC and Fig. 28 for HFGMC. The experimental values are shown as open circles and squares while the four different subcell failure criteria are shown with various line types. It is noted that, for a $\pm 45^{\circ}$ laminate subjected to $\sigma_{\mathrm{y}} / \sigma_{\mathrm{x}}=$ $0 / 1$, there is no theoretical distinction between the $\varepsilon_{\mathrm{y}}$ and $\varepsilon_{\mathrm{x}}$ response. 
The various failure criteria predictions are very similar to one another for both the GMC and HFGMC. All of the failure criteria follow both the $\varepsilon_{\mathrm{x}}$ and $\varepsilon_{\mathrm{y}}$ curve very well, but they all over predict the final failure significantly, by approximately $200 \mathrm{MPa}$.

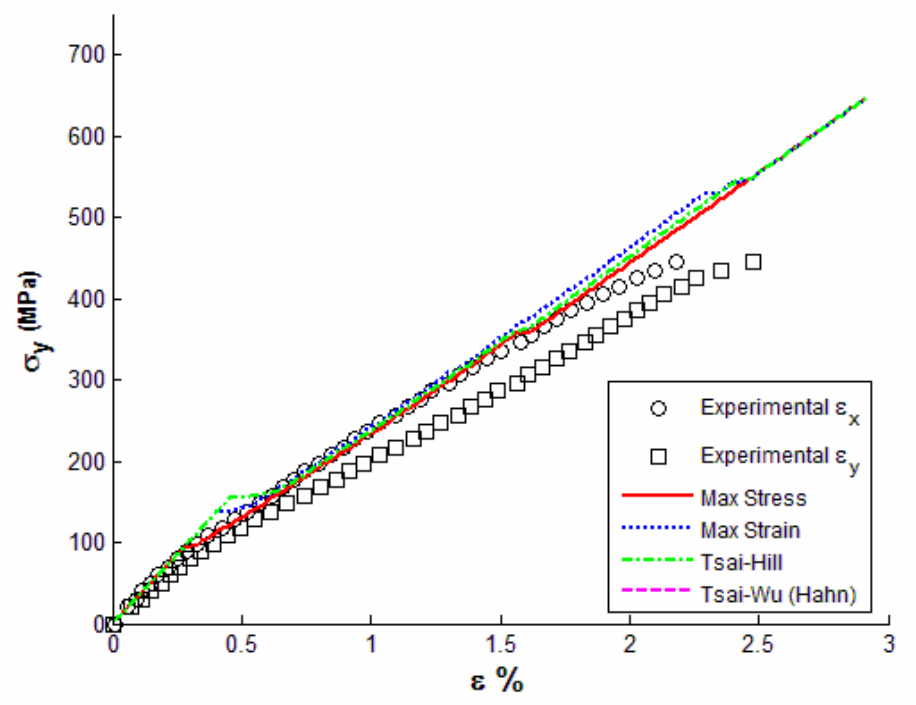

Figure 27. $\pm 45^{\circ}$ angle ply laminate E-glass/MY750/HY917/DY063: Stress/strain curve for $\sigma_{\mathrm{y}} / \sigma_{\mathrm{x}}=1 / 1$ with GMC

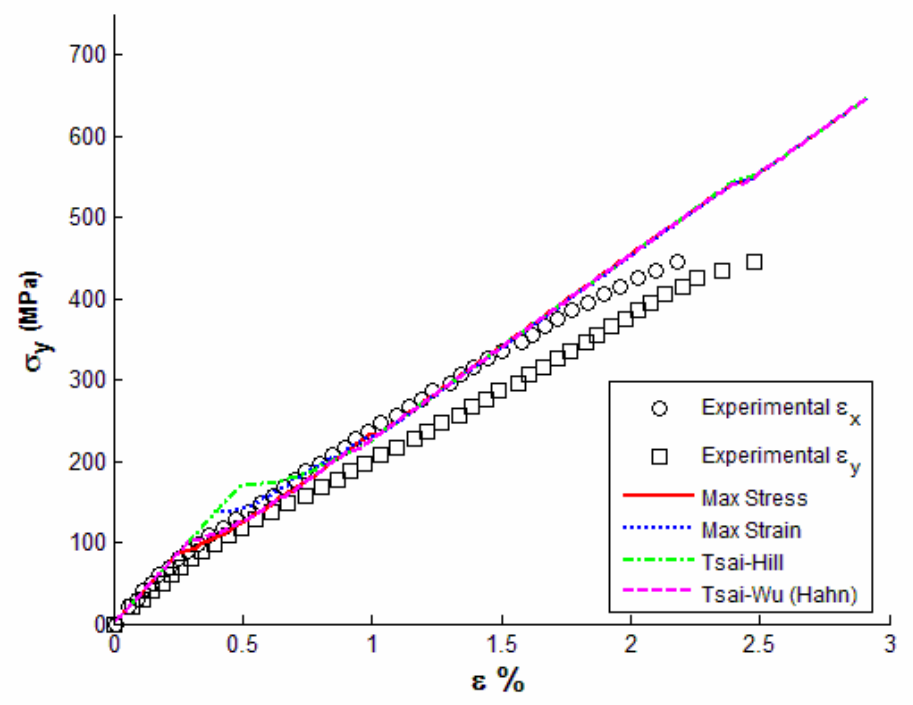

Figure 28. $\pm 45^{\circ}$ angle ply laminate E-glass/MY750/HY917/DY063: Stress/strain curve for $\sigma_{y} / \sigma_{x}=1 / 1$ with HFGMC

\section{E-glass/MY750/HY917/DY063: Stress/strain curve for $\sigma_{y} / \sigma_{x}=1 /-1$}

A comparison of theoretical predictions to experimental results for this load case is shown in Fig. 29 for GMC and Fig. 30 for HFGMC. The experimental values are shown as open circles and squares while the four different subcell failure criteria are shown with various line types.

The various failure criteria predictions are very similar to one another for both the GMC and HFGMC. All of the failure criteria follow both the $\varepsilon_{\mathrm{x}}$ and $\varepsilon_{\mathrm{y}}$ slope very well but they all under predict the final failure significantly, by $50 \mathrm{MPa}$. This case is similar to the $\pm 55^{\circ}$ angle ply laminate E-glass/MY750/HY917/DY063 under the loading ratio 
of $\sigma_{\mathrm{y}} / \sigma_{\mathrm{x}}=1 / 0$, where the ply level strain state is dominated by shear. Once again the models significantly under predict the damage progression prior to final failure.

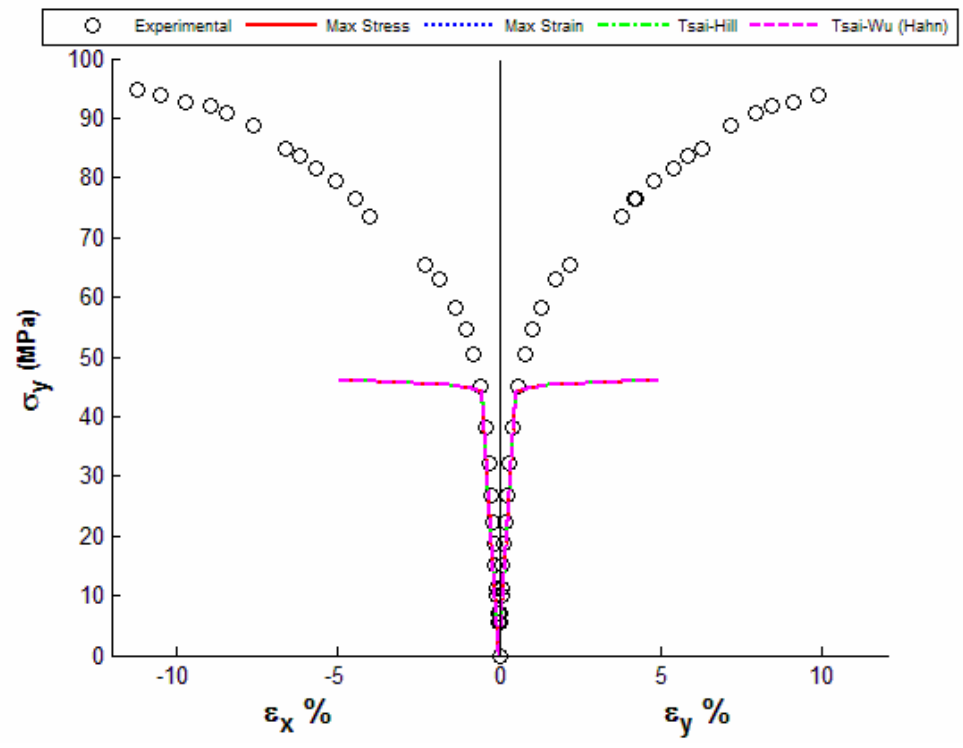

Figure 29. $\pm 45^{\circ}$ angle ply laminate E-glass/MY750/HY917/DY063: Stress/strain curve for $\sigma_{y} / \sigma_{x}=1 /-1$ with GMC

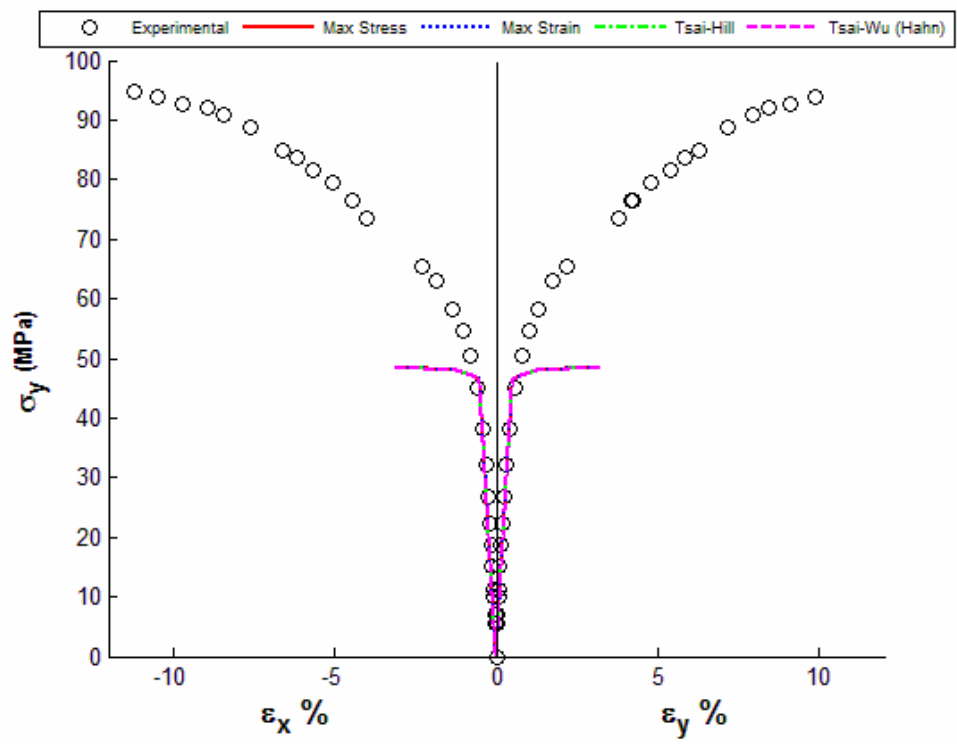

Figure 30. $\pm 45^{\circ}$ angle ply laminate E-glass/MY750/HY917/DY063: Stress/strain curve for $\sigma_{\mathrm{y}} / \sigma_{\mathrm{x}}=1 /-1$ with HFGMC

\section{Conclusion}

The objectives of this paper were to evaluate the current predictive capabilities of the Generalized Method of Cells (GMC) and High-Fidelity Generalized Method of Cells (HFGMC) micromechanics theories for the progressive failure prediction of polymer matrix composite (PMC) laminates and to evaluate the influence of four failure criteria applied at the fiber/matrix constituent scale. Toward this end, these two micromechanics theories were used to model the ply level behavior within classical lamination theory simulations of the Worldwide Failure Exercise (WWFE) data. A comparison among the max stress, max strain, Tsai-Hill, and Tsai-Wu (Hahn) failure criteria was made. It must be stated that this is the first time that GMC and HFGMC have been systematically 
applied to predict PMC laminate failure and that no modifications were made to the fiber/matrix properties provided by the WWFE. The results are thus pure predictions from the models, without the benefit of in-situ property alterations that enable the predictions to match ply level strength data. Also, the simplest damage progression model, a step function, was used.

The results indicate that the choice of failure theory has a significant effect on the predictions, with the Tsai-Wu (Hahn) criterion showing the best agreement with experiment. The differences between the GMC and HFGMC micromechanics theories were small compared to those among the four failure criteria. This is most likely because the HFGMC implementation used average subcell stresses to predict failure rather than quadrature point stresses. Using average stresses negates the benefits of the more accurate stress concentrations provided by HFGMC and makes HFGMC act more like GMC. There is work under way to model damage at the subcell quadrature point level in HFGMC. The results from this paper also show that the predictions match best with the experimental data in cases less dominated by shear at the ply and micro scales. For example, the $\left(0^{\circ} / \pm 45^{\circ} / 90^{\circ}\right)$ AS4/3501-6 laminate predictions (Figs. $13-18)$ and the $\left(0^{\circ} / 90^{\circ}\right)$ E-glass/MY750/HY917/DY063 laminate predictions (Figs. 25 and 26) correlate well with experiment, while the $\pm 55^{\circ}$ E-glass/MY750/HY917/DY063 laminate predictions (Figs. 19 - 24) and even the shear dominated portions of the $0^{\circ}$ lamina predictions (Figs. $3-6$ ) do not. There is thus a clear need for an improved damage progression model that enables a more gradual transition between failure initiation and final failure at the fiber/matrix scale, especially in cases that are dominated by local shear. The overall tendency was for the predictions to be more conservative compared to experimental failure data the more the local behavior was influenced by shear. This again points to the need for a more progressive damage model in shear that enables the dissipation of greater amounts of energy prior to final failure. Work is currently underway to address this need.

\section{Acknowledgments}

The first and second authors gratefully acknowledge the support of this research by NASA grant NNX07AD70A.

\section{References}

[1] Hinton, M. J., and Soden, P. D., "Predicting failure in composite laminates: background to the exercise," Composites Science and Technology, Vol. 58, No. 7, 1998, pp. 1001-1010.

[2] Soden, P. D, Hinton, M. J., and Kaddour, A. S., "Lamina properties, lay-up configuration and loading conditions for a range of fibre reinforced composite laminates," Composites Science and Technology, Vol. 58, No. 7, 1998, pp. 1011-1022.

[3] Soden, P. D, Hinton, M. J., and Kaddour, A. S., "A comparison of the predictive capabilities of current failure theoris for composite laminates," Composites Science and Technology, Vol. 58, 1998, pp. 1225-1254.

[4] Soden, P. D, Hinton, M. J., and Kaddour, A. S., "Biaxial test results for strength and deformation of a range of E-glass and carbon fibre reinforced composite laminates: failure exercise benchmark data," Composites Science and Technology, Vol. 62, 2002, pp. 1489-1514.

[5] Herakovich, C. T., Mechanics of Fibrous Composites, John Wiley \& Sons, Inc., New York, 1998.

[6] Jones, R. M., Mechanics of Composite Materials, Hemisphere, New York, 1975.

[7] Bednarcyk, B. A., and Arnold, S. M., MAC/GMC 4.0 User's Manual, NASA/TM-2002-212077, 2002.

[8] Aboudi, J., "Generalized Effective Stiffness Theory for the Modeling of Fiber-Reinforced Composites," Int. J. Solids Struct., Vol. 17,1981, pp. 1005-1018.

[9] Aboudi, J., Mechanics of Composite Materials: A Unified Micromechanical Approach, Elsevier, Amsterdam, 1991. 
[10] Paley, M., and Aboudi, J., "Micromechanical Analysis of Composites by the Generalized Cells Model," Mech. Materials, Vol. 14, 1992, pp. 127-139.

[11] Aboudi, J., "Micromechanical Analysis of Thermoinelastic Multiphase Short-Fiber Composites," Compos. Eng., Vol. 5, 1995, pp. 839-850.

[12] Aboudi, J., "Micromechanical Analysis of Composites by the Method of Cells - Update," Appl. Mech. Rev., Vol. 49, 1996, pp. S83-S91.

[13] Pindera, M. J., and Bednarcyk, B. A., "An Efficient Implementation of the Generalized Method of Cells for Unidirectional, Multi-Phased Composites with Complex Microstructures," Composites Part B, Vol. 30, 1999, pp. $87-105$.

[14] Bednarcyk, B. A. and Pindera, M. J., "Inelastic Response of a Woven Carbon/Copper Composite Part II: Micromechanics Model," J. Composite Materials, Vol. 34, 2000, pp. 299-331.

[15] Aboudi, J., Pindera, M. J., and Arnold, S. M., "Linear Thermoelastic Higher-Order Theory for Periodic Multiphase Materials," J. Applied Mech., Vol. 68, 2001, pp. 697-707.

[16] Aboudi, J., Pindera, M. J., and Arnold, S. M., "Higher-Order Theory for Periodic Multiphase Materials with Inelastic Phases," Int. J. Plasticity, Vol. 19, 2003, pp. 805-847.

[17] Aboudi, J., "The Generalized Method of Cells and High-Fidelity Generalized Method of Cells Micromechanical Models - A Review," Mech. Adv. Materl. Struct. Vol. 11, 2004, pp. 329-366.

[18] Tsai, S.W., "Strength theories of filamentary structures," in Fundamental Aspects of Fiber Reinforced Plastic Composites (R.T. Schwartz and H.S. Schwartz, eds.), Wiley Interscience, New York, 1968, pp. 3-11.

[19] Tsai, S.W. and Wu, E.M., "A General Theory of Strength for Anisotropic Materials," Journal of Composite Materials, Vol. 5, 1971, pp. 58-80.

[20] Tsai, S.W. and Hahn, H.T., Introduction to Composite Materials, Technomic Pub. Co., 1980.

[21] Bogetti, T. A., Hoppel, C. P. R., Harik, V. M., Newill, J. F., and Burns, B. P., "Predicting the nonlinear response and failure of composite laminates: correlation with experimental results," Composite Science and Technology, Vol. 64, 2004, pp. 477-485. 